\title{
Zur Kenntnis des Wesens der Periarteriitis nodosa.
}

(Aus dem Pathol. Institut der Universität Strabburg. FrühererDirektor: Prof.J. G. Mönckeberg).

Von

Dr. Paul Spiro, früherer Assistent des Instituts.

Obwohl ein halbes Jahrhundert vergangen ist seit der Zeit, da Kußmaul and Maier zum erstenmal das Krankheitsbild der Periarteriitis nodosa beschrieben haben, und obwohl seither fast ebensoviel Fälle von Periarteriitis nodosa veröffentlicht wurden, als Jahre vergangen sind, herrscht noch heate ein völliges Dunkel über das Wesen der Erkrankung, ein Dunkel, das es verständlich erscheinen läßt, wenn fast jeder Autor, der einen Fall von Periarteriitis nodosa mitteilt, im Laufe seiner Ausführungen za dem Schluß kommt. daß jeder Fall eo ipso als der Publikation würdig erachtet werden muß und als geeignet angesehen werden kann, Licht in dieses Dunkel zu bringen.

Es sei darum gestattet, im folgenden über einen Fall von Periarteriitis nodosa zu berichten, der am 12. September 1917 im Pathologischen Institut der Universität Straßburg zur Obduktion gelangte.

Aus der weitläufigen Krankengeschichte des K. G., 23 Jahre alt, Arbeiter, seien folgende Daten entnommen:

Familien-Anamnese o. B.

Patient selbst will früher nie ernstlich krank gewesen sein, leugnet im besonderen jegliche venerische Infektion. Seit Februar 1915 litt er an immer wiederkehrenden Geschwüren, besonders an beiden Armen und Beinen; seither befand er sich fast ständig in verschiedenen Lazaretten des Feldheeres, sowie in Truppenkrankenstuben, wo er überall auf Furunkulose behandelt wurde. Deswegen wurde er am 22. Oktober 1916 in ein Feldlazarett einer Landwehrdivision überwiesen. Von dort am 25. Oktober 1916 wegen einer nie ganz zur Ausheilung gekommenen Krätze einer Krankentransportabteilung, von dort wiederum der Leichtkrankenabteilnng eines Etappenlazaretts überwiesen. Daselbst konnten am Allgemeinbefund keinerlei Veränderungen nachgewiesen werden; im speziellen wurden aber auch hier Haatulzerationen gefunden, die sich nach Verbänden - mit essigsaurer Tonerde - reinigten, so daß Patient am 18. November 1916 als geheilt zur Truppe entlassen wurde.

Am 20. Dezember 1916 wegen "Narbe am Glied" Blutentnahme zur Wassermann-Probe; Wassermann positiv, deshalb am 8.1.1917 einem „Festungslazarett, speziell für Haut und Geschlechtskrankheiten" überwiesen. Daselbst wurden "Krätze" und

Yirchows Archiv f. pathol. Anat. Bd. 227, Heft 1. 
"Lues latens" als spezieller Befund festgestellt, während sich am Allgemeinbefund keine Veränderungen nachweisen ließen.

Vom 9.1.17-12.1.17 mit solutio Vleminghx behandelt.

Ab 13. 1. 17 antiluetische Kur.

13.1. $17=0,1 \mathrm{Hg}$. salicyl. intergluteal.

15. $1.17=0,1 \mathrm{Hg}$. salicyl. interghtreal.

18. $1 \cdot 17=0,3$ Neosalvarsan intravenös.

23.1.17 nach Hg.-Injektion keinerlei Beschwerden.

26. 1.17 bringt bei der Visite keinerlei Klagen vor.

30. 1.17 nach Hg. keine Beschwerden.

3. 2. 17 Allgemeinbefinden $0 . \mathrm{B}$.

8. 2.17 auf Befragen keine Klagen.

10. 2. 17 hat Hg.-Injektion angeblich diesmal nicht gut vertragen, nur Kopfschmerzen, sonst o. B.

13. 2.17 nach Salvarsan keinerlei Störung.

14. 2. 17 Albumen $=0$.

16. 2.17 im Allgemeinbefinden keinerlei Störung.

18. 2.17 klagt über etwas Kopfschmerzen.

20. 2. 17 nach Hg. keinerlei Beschwerden, nur etwas angegriffen, etwas Kopfschmerzen.

23.2. 17 auf Befragen heute keine Beschwerden.

26. 2. 17 Befinden in Ordnung.

6. 3. 17 Kur beendet. Symptome- und beschwerdefrei, als erwerbsfähig und k. v. entlassen (zur Truppe!).

Am 11. 5. 17 meldete sich Patient wegen Schmerzen im Rücken; in den Seiten und Kniegelenken, sowie wegen Schwächeanfälle krank, wurde, wiederum einem zur Behandlung von Haut- und Geschlechtskrankheiten eingerichteren Festungslazarett überwiesen („zur Beobachtung auf gastrische Krisen nach Lues").

14. 0. 17 Blutentnahme zum "Wassermann". "Wassermann" ergibt schwache, partielle Hemmung der Hämolyse, also "verdächtig". Körpergewicht $64 \mathrm{~kg}$.

Im Festungslazarett wird am 15.5. 17 der Allg emeinbefund als o. B. bezeichnet; als spezieller Befund: Lues latens diagnostiziert.

Daselbst alsdann folgende Therapie.

Neo-salvarsan

1. am 22. 5. $17=0,3$

Temperat. abds. 36,6

" morg. 37,0

2. $\operatorname{am} 30.5 \cdot 17=0,45$

Temperat. abds. $36.6 ; 36,0$

morg. 36,2

3. $\operatorname{am} 6 \cdot 6 \cdot 17=0,6$

Temperat. nachm. 36.8

$$
\text { " abds. } 36,5
$$

Körpergewicht

18. 5. $17=0,05$

21. $5.17=0,1$

24. 5. $17=0,1$

29. 5. $17=0,1$

1. $6.17=0,1$

4. $6 \cdot 17=0,1$

8. $6 \cdot 17=0,1$

11. $6 \cdot 17=0,1$

14. $6.17=0,1$
$25.5 .=63 \mathrm{~kg}$

25. $5 .=63$ "

1. $6 .=60$ "

9. $6 .=59$,

15. $6 .=56 "$

21. $6 .=55$

29. 6. $=54,5$.

Am 17. 5. xhenmatische Schmerzen in beiden Schultern.

Am 18. 5. Albumen $=0$. Auf Leistendrüsen beiderseits Jodkalisalbe, Einreiben des

Rückens an den schmerzenden Stellen mit Chloroformöl, täglich 3 mal 1 Aspirin à $0, \tilde{y}$.

22. 5. kein Albumen.

23. 5. rheumatische Schmerzen gebessert.

25. 5. kein Albumen.

26. 5. Schmerzen im linken Schultergelenk, 3 Aspirintabletten. 
29. 5. Schmerzen im linken Schultergelenk, 6 Aspirintabletten.

30. 5. Albumen $=0$.

31. 5. Schmerzen im Schultergelenk nach Angabe des Patienten fast verschwunden.

1. 6. kein Albumen.

4.6. Einreiben der Schultern, wo wieder etwas Schmerzen, mit Kampferspiritus.

6. 6. kein Albumen.

8. 6. Schmerzen in Schultergelenk gebessert.

9. 6. kein Albumen.

12. 6. rheumatische Beschwerden völlig verschwunden.

14. 6. leichte Stomatitis. Einpinseln mit Chromsäure.

15. 6. Spuren von Albumen: Bettruhe, Diät, heiße Bäder.

16. 6. " " antisyphilitische Kur abgebrochen.

19.6. " " Milchdiät, heiße Bäder, Bettruhe, Blutentnahme zum Wassermann.

Wassermann negativ. Hämolyse vollständig.

25. 6. Temperatur morgens 37,2; Patient klagt über Leibschmerzen, Leib nicht druckempindlich; Fortsetzung der Milchdiät, Spuren von Eiweib.

27. 6. in Urin Spuren Albumen, im Sediment Leukozyten, keine Zylinder.

28. $6 .$,

2. 7. " , geringe Mengen Albumen.

3. 7. " " geringe Mengen Albumen, geringe Temperaturschwankungen (zwischen 37,2 und 37,8 ). In den letzten Tagen Koliken, am 3.7. besonders stark. Wird zur Weiterbehandlung der Albuminurie nach einem „Festungslazarett für innere Krankheiten“ verlegt.

Daselbst folgender Befund; R. H. O. an den Lungen hauchendes Exspirium; 2. Ton an der Basis des Herzens gespalten, sonst alles o. B., auch Reflexe o. B.

Urin: Tagesmenge $=2 \mathrm{l}$; spez. Gewicht 1014; Albumen $=0 ;$ Zucker $=0$.

5. 7. Pat. Klagt über Schmerzen im Unterleib, kein objektiver Befund.

8. 7. Schmerzen nachgelassen! Therapie: warme Wickel.

14. 7. keine Änderung.

19. 7. Urin: Albumen -, Saceh .-.

31. 7. immer noch matt und elend, objektiv nichts Neues. Ordination: Natr. bic. Xatr. phosphat.

7. 8. Weniger Beschwerden im Abdomen; aber sehr matt, objektiv nichts.

14. 8. keine Änderung! Urin frei von Alb., Sacch.

16. 8. in der Nacht vom 15./16.8. epileptische Anfälle vom wachhabenden Arzt beobachtet: Nach dem Anfall Motilität nicht gestört; Sensibilität vielfach gestört, Kornealreflex fehlt: Patellarreflex lebhaft; beiderseits dentlicher FuBklonus. Während des Anfalls keine Krämpfe. Schanm vor dem Munde, kein Zungenbiß, vollständige Bewußtlosigkeit. 16. 8. Pat. hat einen fünften Anfall - wie die vorhergehenden -, kommt nach 25 Minuten wieder zu sich, weiß von nichts, will früher nie Anfälle gehabt haben.

Nach einem "Festungslazarett für Nervenkranke" verlegt mit der Diagnose: Ka chexie nach Lues, Hysteroepilepsie.

Im übrigen geht die Krankengeschichte weiter:

16. 8. im Lauf des Nachmittags fünf neue Anfälle. In den anfälsfreien Augenblicken stark benomnen, unverständliche Worte vor sich hinmurmelnd.

Körperlicher Befund o. B.

Nervensystem: Achillessehnenreflex bds. gesteigert. Patellarsehnenreflex bds. gesteigert. Kein Babinski, kein Fußklonus.

Bis 21. 8. anhaltende Besserung, nur immer große Mattigkeit and Benommenheit. (Gibt hier anamnestiseh an. daß er seit seiner Kindheit an geschwollenen Leistendrüsen 
gelitten habe, gibt ferner auf spezielles Befragen an. daß er verheiratet sei und ein gesindes Kind habe.)

Ab 27. 8. zunehmend apathisch, hinfällig; Abdomen kahnförmig eingezogen. Lumbalpunktion ergibt klare Flüssigkeit. EiweiBreaktion nach Kochprobe nicht vermehrt: ausgesprochene Lymphozytose; "Wassermann" im Liquor negativ.

In der Nacht vom 27.8. zum 28. 8, wieder fünf epileptische Anfälle.

28. 5. zunehmende Verschlechterung des Allgemeinzustandes. hinfalliger Ausdruck in den eingefallenen Gesichtszügen: Abdomen stark eingezogen.

Achillessehnenreflex und Patellarsehnenreflex beiderseits sehr lebhaft; "Babinshi" beiderseits vorhanden; "Kerning" dentlich positiv, ausgesprochene Nackenstarre; Pus klein, regelmäßig; Temperatur normal. Nach der Untersuchung entleert sieh aus der Hararöhre massenhaft gelblich trübe Flüssigkeit, massenhaft Lenkozyten, keine Spermatozoen. keine Gonokokken. Sonst kein körperlichor Befund, keine Druckempfindlichkeit des Abdomens.

30. 8. Pat. geistig wieder klar, sehr matt und hinfällig, nach der UniversitätsNervenklinik verlegt (Diagnose: Lues cerebri).

Universitäts-Nervenklinik:

Aufgenommen 30. 8. Pat. klagt über allgemeine Mattigkeit und Schmerzen im ganzen Körper. Befund 0. B.

31. 8. Allgemeinbefund o. B. Druckempfindlichkeit in der rechten Unterbauchseite, mehrmals breiige, mit Blut untermischte Durchfälle; kein spontanel Urinabgang. Reflexe lebhaft; kein Klonus; „Babinski“ rechts ++ , links -: Sensibilität nicht gestört, große Nervenstämme überall etwas druckempfindlich. Motilität: keine Lähmungen, keine Spasmen.

Bis 5. 9. Durchfälle, die auf Opiam stets etwas nachlassen. Im Status nervosus und Status psychicus leichte Besserung. Stuhluntersuchung auf Typhus und Dysenterie negatix. Am rechten Oberarm ein schmierig belegter, 1-Pfennigstück großer Substanzdefekt.

7.9. schlechte Nahrungsaufnahme, klagt stärker über allgemeine Schmerzen.

8. 9. starke Klagen über Kopfschmerzen, unwillkürlicher Stuhlabgang, schmierig belegter Defekt am rechten Zungenrand.

In der Nacht vom 8.-9.9. starke Schwellung der rechten Parotis. LäBt Stubl und Urin unter sich. „Babinski" bds. ++ ; "Oppenheim" bds. ++ ; Hypotonie der Muskulatur.

10.9. Schwellung der Parotis zurückgegangen, sonst Status unverändert.

12. 9. morgens 8 Uhr Eixitus letalis.

\section{Die Sektion ergab folgenden Befund.}

$173 \mathrm{~cm}$ großer, kräftig gebauter männlicher Körper in mäßjigem Ernährungszustand. mit gut ansgebildeter Muskulatur.

Außere Haut: mäBig blab, frei von Exanthemen und Odemen; sichtbare Schleimhänte blaß.

Pupillen: gleich weit, mittelweit, rund.

Natürliche Körperöffnungen: 0 . B.

Unterhautzellgewebe: mäßig reduziert.

Muskulatur: gat entwickelt.

Die Bauchdecken eingesunken; nach Eröffnung der Bauchhöhle zeigt sich. $\mathrm{dab}$ die Bancheingeweide in regelrechter Lage liegen; das Peritoneum glatt, fettarm, frei von pathologischen Veränderungen; die Darmschlingen mäßig gebläht; speziell am Processus vermiformis keinerlei Verwachsungen, keine Injektion.

$Z$ werchfellstand links oberhalb der 5 , rechts an der 4 . Rippe. 
In der Brusthöhle regelrechter Situs der Brusteingeweide. In den beiderseitigen Interpleuralräumen keinerlei Verwachsungen, keinerlei Flüssigkeit.

Der Herzbeutel liegt in ungefähr Handbreite frei; im Herzbeutel geringe Menge klarer, seröser Flüssigkeit.

Die Größe des Her zens entspricht der Körpergröße; das Epikard im allgemeinen glatt, mit spiegelnder Oberfläche, nur an einzelnen Stellen vorgebuckelt durch eigentümliche Knotenbildungen: Diese Knotenbildungen sind sowohl auf der Vorderfläche als auch anf der Rüekfläche des Herzens anzutreffen, sowohl über dem linken als auch über dem rechten Fentrikel, aber durchweg im Verlanfe der KranzgefäBe, und zwar der kleineren Äste der Koronararterien zu finden, die gleichsam von ihnen umseheidet erseheinen: sie sind im allgemeinen linsengroß, erreichen vereinzelt die Größe eines Haselnußkerns, sind gelblich weiß und von fester Konsistenz. Sie sind auf dem Durchschnitt durchweg von blutroter Färbung und von einem ganz schmalen, anscheinend aus weißlichem Bindegewebe gebildeten Rand umgeben; das Zentrum scheint auf einzelnen Schnittflächen direkt mit einem ehenfalls blutroten Pfropf im Lumen dés Arterienastes, dem sie anlagern, zusammenzuhängen.

Das Myokard des Herzens ist von Fleischfarbe, fester Konsistenz, durchweg frei von Schwielenbildungen und frei von Erweichungsherden.

Das Endokard ist überall glatt und zart, der Klappenapparat intakt; die Höhlen des rechten Herzens sind entsprechend weit, die Wandung des rechten Vorhofs und des rechten Ventrikels überall entsprechend dick; auch im linken Vorhof zeigen sich keine pathologischen Veränderungen in der Weite des Lumens und in der Dicke der Wandung, hingegen erscheint der linke Ventrikel leicht erweitert, die Wandung deutlich, wenn auch nicht sehr hochgradig verdickt; Gewicht des Herzens $335 \mathrm{~g}$.

An den größeren Ästen der Kranzgefäßen ist die Intima - soweit die KranzgefäBe bei der Sektion geöfnet werden - glatt und zart, das Lumen nicht verengt, für eine $2 \mathrm{~mm}$ Sonde gut durchgängig.

Die Intima der Aorta und Pulmonalis ist glatt und spiegelnd, ihre Weite ist entspreehend.

Die Pleurablätter sind durchweg glatt und spiegelnd.

Die Lungen sind reichlich lufthaltig, mäBig saftreich, mit starkem, in den unteren Partien hypostatisch gesteigertem Blutgehalt, aber frei von jeglichen Herdbildungen. An den Langenarterien lassen sich keinerlei Veränderungen nachweisen. Die großen Bronchen sind frei von Veränderungen.

Peribronchiale und mediastinale Lymphknoten stark anthrakotisch, sonst 0 . B.

An den Halsorganen kein besonderer Befund.

Milz 12\%: $: 8: 3 \mathrm{~cm}$, dunkelrot, derb, mit sehr deutlich gezeichnetem Parenchym. Thre Kapsel überall straff gespannt, frei von Verdickungen.

Nebennieren o. B.

Nieren von mittlerer Größe, links 180 , rechts $160 \mathrm{~g}$ schwer, ihre Kapseln beiderseits leicht abziehbar. An beiden Nierenoberflächen finden sich zahlreiche, strahlig eingezogene, konsistente Partien, die durchschnittlich ungefähr haselnußgroß, deutlich abgegrenzt und gelblich gefärbt, aber in ihrer ganzen Peripherie von einer schmalen, ungefähr $1 \mathrm{~mm}$ breiten, donkelroten Randzone nmgeben sind; beim Durchschneiden der Niere zeigt sich; daß diese Herde kegelförmig sich verjüngend in die Rinde eindringen und an der Grenze von Marksubstanz and Rindensubstanz endigen. In beiden Nieren finden sich, durch das ganze Parenchym zerstreut, außerdem zahlreiche, scharf umgrenzte, von einer ganz schmalen, weißen Kapsel umgebene Blutherde, die zumeist in unmittelbarer Nachbarschaft und, wie es scheint, auch in unmittelbarer Kommunikation neben kleinsten Nierengefäßen liegen. Die Rindensubstanz beider Nieren erscheint auffallend blaß und hebt sich scharf von der dunklen Marksubstanz ab, über deren Niveau sie überall ödematös gequollen emporragt. Die Marksubstanz selbst erscheint an beiden Nieren unverändert; Nierenbecken und Nierenhilus, sowie Ureteren o. B. 
Die Blasenschleimhaut im allgemeinen glatt und weib, von einzelnen ungefähr apfelkerngroßen Blutungsherden durchsetzt. Die Wandung der Blase nicht verdickt, ihre Muskulatur nicht verstärkt. An der Pars prostatica der Uxethra keine Veränderungen. Prostata und Samenbläschen ohne abnormen Befund. Hoden, Nebenhoden, Samenstrang ebenfalls o. B., desgleichen die Arteriae spermaticae.

Die Leber zeigt an ihrer Oberfläche, speziell an der unteren Kante, mehrere ungefähr kirschkerngroße, strahlig eingezogene Partien, in denen das Leberparenchym weibgelb, frei von jeglicher Zeichnung erscheint. Diese Partien setzen sich, an Größe zunehmend, in das Innere der Leber fort, unregelmäßig geformt, aber überall scharf gegen das umgebende Parenchym abgegrenzt nnd überall von einer dentlichen. ungefähr $1 \mathrm{~mm}$ breiten, dunkelroten Randzone umgeben. Im Inneren der Leber finden sich in Strängen der Glis s o n schen Kapsel liegend, periarterielle Knotenbildungen, deren Inneres von dunkelroten, fast schwärzlichen, konsistenten Blutgerinnseln erfült ist und deren Wandung von einer ganz schmalen, oft nicht deutlich sichtbaren, narbig weißen Bindegewebszone gebildet wird. Im übrigen ist die Leber von mittlerer Größe, $2190 \mathrm{~g}$ schwer, von gelblich-graner Färbung und mit deutlicher, aber nicht pathologisch gesteigerter Läppehenzeichnung. Am Gallenapparat keine pathologischen Veränderungen.

Portale Lymphknoten o. B.

Am Magen kein abnormer Befund, ebenso am Pankreas.

An den Mesenterialgefäßen lassen sich keine Veränderungen nachweisen: ihre Innenfläche ist überall glatt und spiegelnd, ihr Lumen nirgends verändert.

Die Darm-Serosa ist überall intakt; die Darmschleimhant ist im Duodenum und im Jejunum frei von Ulzerationenen, frei von Injektionen, ohne Schwellungen des FollikelApparates. Hingegen ist am oberen Lleum, scharf nach beiden Seiten hin abgegrenzt, eine Schleimhautpartie von ungefähr $25 \mathrm{~cm}$ Länge, auffallend gerötet und geschwollen, vielfach auch von zackig umrandeten, unregelmäßig geformten Ulzerationen mit blatig belegtem Grunde durchsetzt. Im unteren Ileum ist die Schleimhaut wiederum völlig intakt, ebenso im Coecum und im Colon ascendens. Hingegen beginnen in Colon transversum wiederum scharf abgegrenzte Veränderungen der Schleimhaut, die sich bis zum Anus erstrecken. Hier ist die Sehleimhaut durch zahlreiche, sehr flache, überall nur bis in die Submukosa und nirgends bis an die Muskularis herangreifende Defekte, die unregelmäßig an Größe und Form vielfach miteinander konfluieren, in breiter Ausdelnung zum Verschwinden gebracht. Vereinzelt erhaltene Schleimhautinseln sind dnnkel pigmentiert, ödematös geschwollen, vielfach blutig belegt; Schleimhautbrückenbildungen finden sich aicht.

Bewegungsapparat $0 . B$

Gehirn un'd Rückenmark werden zunächst in toto in Formol eingelegt und nach acht Tagen seziert: dabei finden sich weder im Gehirn noch im Rückenmark pathologische Veränderungen.

\section{Mikroskopische Untersuchung.}

Es werden sieben Nierenstücke, sieben Herzstücke und fünf Leberstücke, die die erwähnten. eigentümlichen Knotenbildungen enthalten, ferner fünf Darmstücke ans den veränderten Partien und beide Arteriae vertebrales and Arteriae fossae Sylvii in $10 \%$ oiger Formollösung gehärtet und nach Paraffineinbettung geschnitten. Sämtliche Präparate werden mit Lämatoxylin-Eosin, nach Weigert-yan Gieson und nach Weigerts Methode der ElastikaFärbung gefärbt, fernerhin nach Löffler auf Bakterien, nach Koch-Ehrlich auf Tuberkelbazillen und nach Levaditi auf Spirochäten untersucht. 
Niere: Fast sämtliche Schnitte zeigen die gleichen Bilder: ein arterielles Gefäß auf seiner ganzen Zirkumferenz von im wesentlichen intakter Wandung, d. h. völlig unveränderter Adventitia, absolut normaler Media, durchaus unveränderten Elasticae und leichtgewucherter Intima umgeben; dicht daneben ein das Gefäßlumen an Ausdehnung weit übertreffender, vielfach schon in Organisation, sogar schon in Kanalisation stehender Blutherd, nach allen Richtungen hin von derbem, kernarmem Bindegewebe begrenzt.

Im speziellen konnte in allen Bildern gleichermaßen konstatiert werden, daß die Adventitia der Arterien frei von Infiltration ist, daß in der Media keinerlei Anzeichen einer regressiven Metamorphose zu erkennen sind, daß die Elasticae leicht geschlängelt verlaufen, und daß weder an der Elastica interna noch an der Elastica externa eine Zerreißung oder etwa eine Aufsplitterung der elastischen Lamellen zu sehen ist, daß die Wucherung der Intima aus einem kernreichen, faserigen, retikulär angeordneten Bindegewebe besteht, an dem sich keinerlei Spuren irgendeiner Neubildung von elastischen Fasern, aber auch keine Spuren irgendeiner Entartung, etwa einer hyalinen Degeneration oder einer Verfettung, erkennen lassen. Bez. des der Arterie anliegenden Blutherdes konnte in allen Bildern gleichermaßen festgestellt werden, daß die Organisation und Kanalisation von der bindegewebigen Kapsel gleichsam konzentrisch ausgeht, während im Innern noch unberührt zusammengesinterte rote Blutkörperehen liegen.

Die Vermutung, die sich bei derartigen Bildern aufdrängt, daß zwischen den Arterien und den in ihrer unmittelbaren Umgebung liegenden Blutherden ein Zưsammenhang bestehe, wird durch zwei spezielle Bilder bestätigt:

In dem einen Bild sieht man die Arterie, schräg getroffen, sich plötzlich kelchartig erweiternd, um sich ganz abrupt in ein sackartiges Aneurysma auszubuchten, das von gleichmäßigen, von den Rändern her in beginnender Organisation begriffenen Blutmassen erfüllt wird. Bis zur Ausbuchtnngsstelle ist die Gefäßwand intakt, das Gefäßlumen aber anscheinend erfüllt von einem zarten, kernreichen Granulationsgewebe, das kontinuierlich einerseits in die ihres Endothels beraubte, leicht gewueherte Gefäßwandintima, andererseits in die Randpartien der Anenrysma-Blutmassen übergeht. An der Ausbuchtungsstelle reißen die mittleren Partien der Gefäßwand, also Elastica interna, Media, Elastica externa plötzlich ab, während die gewucherte Intima in das den Rand des Thrombus bildende Granulationsgewebe ausläuft und die Adventitia direkt in das die Blutmasse begrenzende, derb fibröse Narbengewebe übergeht.

In dem andern Bild sieht man ein Gefäßlumen, auf der einen Seite begrenzt von stark gewucherter Intima, über der hutförmig ein Strang völlig intakter, aber an beiden Enden selarf abgerissener Media sitzt, daran Stränge der leicht wellig verlaufenden, an beiden Rändern abrupt abgerissenen Elasticae und eine an den beiden Enden unscharf begrenzte Adventitia, auf der andern 
Seite begrenzt von Thrombusmassen, die ein Ring derb fibrösen Bindegewebes abschliebt.

Herz: Stück 1: Kleines epikardiales Gefäß. Auf der einen Seite begrenzt von im wesentlichen normaler Gefäßwand, bestehend aus intakter Ad ventitia, intakter Media, intakten Elasticae und nur leicht gewucherter Intima; auf der andern Seite begrenzt von Thromben, die gegen das umgebende Epikard von einer Kapsel derb fibrösen und völlig indifferenten Bindegewebes abgeschlossen sind, aus der heraus aber ein kernreiches, jugendliches Granulationsgewebe, von Blutgefäßen durchzogen, in die Thrombusmassen einwächst.

Auf Stufenschnitten fortschreitende Veränderungen in dem gleichen Sinne; der Thrombus wächst, übertrifft schließlich das Gefäßlumen um ein bedeutendes an Umfang, bleibt aber ständig auf der einen Seite des GefäBes liegen. während sich auf der andern Seite intakte Gefäßwand ausdehnt.

Stück 2: zeigt verschiedene kleine Gefäße mit im wesentlichen intakter, nur durch eine leichte Wucherung der Intima ausgezeichneter Wandung, in ihrer Umgebung aber größere Blutherde, die teilweise schon in Organisation. teils sogar schon in Kanalisation begriffen und von derb fibrösem Bindegewebe abgekapselt sind.

Ebenso Stück 3 .

Auch Stüek 4 zeigt ein entsprechendes Bild: ein arterielles Gefäß; auf der einen Seite begrenzt von einer durch alle Schichten hindurch im wesentlichen intakten, wiederum nur durch eine Wucherung der Intima abgegrenzten Wandung; auf der andern Seite begrenzt von in Organisation begriffenen, pyramidenförmig gegen das Gefäßlumen vordringenden Thrombusmassen.

Auf Stufenschnitten zeigt sich, daß die thrombotischen Massen einerseits ganz plötzlich an Ausdehnung stark gewinnen, andererseits sich plötzlich darin elastische-Fasern finden, die senkrecht zum GefäBlumen verlaufen und rom Gefäßlumen aus auseinanderweichen: wohl ein Zeichen dafür, daß die Gefäß-Ruptur; die zu der hier im mikroskopischen Bilde sichtbaren AneurysmaBildung geführt hat, an einer Gefäßteilungsstelle eingetreten ist.

Auch Stück 5 zeigt ein Gefäß, das auf der einen Seite von im wesentlichen intaktel Gefäßwand, auf der andern Seite von fibrös abgekapseltem Gerinnsel abgegrenzt wird.

Ebenso Stüek 6 .

Stück 7 zeigt ein ganz typisches Bild: In den obersten Sehnitten einer Serie erblickt man einen von derben Bindegewebszügen abgekapselten Blutherd. daneben ein völlig intaktes GefäB. Auf tiefer liegenden Stufenschnitten verändert sich das Bild, indem die Kommunikation zwischen Gefäß und Blutextravasat deutlich wird: man sieht, wie das Gefäß schräg, fast längs getroffen, fast in sämtlichen Tiefen seiner Zirkumferenz durch alle drei Wandschichten hindurch völlig intakt, bezw. nur durch eine starke Intima-Wucherung verändert, an einer schmalen Stelle scharf durchbrochen wird, aus der nun, 
wie ein Flüssigkeitstropfen aus einer Kanüle, Blatmassen herauszuquellen scheinen, die auf allen Seiten umgeben sind von derbem Bindegewebe, in das aus der stark proliferierenden Intima Züge kernreichen Granulationsgewebes fächerförmig einstrahlen. Auf den tiefsten Stufenschnitten gelangt man alsdann in Lagen, in denen das zerstörte Gefäß, nunmehr wiederum völlig intakt, bezw. nur durch eine diffuse Intima-Verdickung verändert, neben dem abgekapselten Blutungsherd einherläuft.

Auch an den Herzschnitten konnte festgestellt werden, daß die äußeren Gefäßwandschichten der Adventitia und Media, sowie die beiden Elasticae, soweit sie vorhanden waren, völlig intakt waren, daß die Wucherungen der Intima ans einem jugendlichen, mäßig zellreichen Granulationsgewebe bestanden, in dem sich keinerlei Spuren einer Degeneration, anch keinerlei Anzeichen einer differenzierenden Neubildung, etwa elastischer Fasern, nachweisen ließen.

Leber: Die Arterien der Leber zeigen ganz entsprechende Veränderungen: Die Gefäßwand ist auf der einen Seite, manchmal auch auf Dreivierteln der Zirkumferenz unverändert, d. h. bis auf eine.Wucherung der Intima völlig intakt, während sie auf der andern Seite, manchmal auch nur in einem einzigen Quadranten der Zirkumferenz völlig fehlt, gleichsam hingeschwemmt von Blutmassen, die sich in das umgebende Bindegewebe hineinergossen haben, von dem sie aber wiederum durch einen Wall narbigen Bindegewebes getrennt werden. Zwischen der erhaltenen Seite der Gefäßwand und den Randpartien des Thrombus spannt sich, in den Thrombus hineinwachsend, die Wucherung der Intima aus, die vielfach derart hochgradig ist. daß das Gefäßlumen völlig obturiert erscheint.

Darm: Im Darm finden sich in mikroskopisch kleinen Gefäßen der Submukosa ganz analoge Veränderungen, allerdings mit einer Besonderheit: die Blutmassen, die hier an den Gefäßen lagern, sind durchweg schon organisiert, und zwar stets von einem kernreichen Bindegewebe, das mit der Intima der Gefäßwand in völlig kontinnierlichem Konnex steht, so daß man zu dem Eindruck gelangt, daß die Blutungsherde von erhaltenen IntimaPartien zugehöriger Gefäße ans zur Organisation gebracht worden seien. -

Auch in der Leber und im Darm finden sich nirgends Veränderungen, die für eine typisch regressive oder typisch progressive Metamorphose der Arterienveränderungen sprechen. Nirgends sind Zeichen eines akuten Entzündungsprozesses vorhanden. Einzig and allein in der Umgebung einzelnar Aneurysmen kleinster Leberarterien finden sich ganz geringe, benachbarte Kapillaren oder benachbarte Nerven nmgebende, perivaskuläre oder perineurale Infiltrate, durchweg aus einkernigen Rundzellen, zu einem großen Teil aus Plasmazellen bestehend, also Zeichen eines abgelaufenen oder chronisch gewordenen Entzündungsprozesses.

Aber es finden sich auch nirgends Veränderungen, die es gestatten 
würden, eine Vermutung über die Natur, resp. über eine eventuelle Spezifität des den Aneurysmen zugrunde liegenden Prozesses anzusprechen, nirgends finden sich Tuberkel oder Gummibildungen oder ihre Folgezustände, wie Verkäsungen, Verkreidungen, oder bei Spezial-Färbung Mikroorganismen, durchaus im Einklang mit der Tatsache, daß sich anch im übrigen Organismus keinerlei Zeichen einer Tuberkulose oder einer Syphilis feststellen ließen.

Die von den erkrankten Arterien versorgten Organe zeigten in ganz charakteristischer Ausprägung jene Veränderungen, die auftreten, wenn die arterielle Blutzufuhr unterbrochen wird und kein genügender Kollateralkreislauf zustande kommt, nämlich typische Infarktbildungen und ilre Residuen: im Herzen die Folgezustände myomalazischer Herde, allerdings nur mikroskopisch sichtbare Wucherungen des interstitiellen Bindegewebes, „Cicatrices cordis"; in den Nieren frischere Infarktbildungen mit Koagulationsnekrose, hyperämischer Randzone und einem Hof zerfallender Lenkozyten: desgleichen in der Leber; im Darm starke Nekrosen und ausgedehnte Defekte der Schleimhaut, teilweise auch Bilder, die als eine hämorrhagische Infarzierung der Darmwand zu deuten waren, nämlich hochgradige Füllung der Venen der Submukosa und Blutaustritte in der Muskularis and Serosa. Dabei erreichten solche Veränderungen nur im Darm eine größere Ausdehnung. während sie im Herzen, in der Leber und in beiden Nieren auf kleine Gebiete beschränkt blieben.

An den Gehirngefäßen ließ sich nirgends anch nur die geringste Veränderung nachweisen. -

Nachdem so alle Veränderungen, die sich nachweisen ließen. als Endstadien eines abgelaufenen Prozesses, nämlich als Aneurysmen, speziell als "falsche Aneurysmen" - nach neverer Nomenklatur als nextramurale Hämatome" (Benda) analysiert worden waren, erhob sich die Frage, auf welcher Basis die Veränderungen zustande gekommen seien. Bei den Aneurysmabildungen konnte es sich handeln um:

1. traumatische Aneurysmen,

2. embolische Aneurysmen oder Aneurysma-Bildungen bei Thronboendarteriitis metastatica purulenta (Benda),

3. tuberkulöse Aneurysmen oder Aneurysma-Bildungen bei Periarteriitis tuberculosa,

4. Aneurysma-Bildungen bei Arteriitis syphilitiea,

o. Aneurysma-Bildungen bei Periarteriitis nodosa.

Eine traumatische Genese der Aneurysmen konnte von vornherein ausgeschaltet werden auf Grund der Multiplizität, in der sie auftraten, und auf Grund der Lokalisation, derzufolge sie sich durchweg innerhalb einer röllig intakten Ungebung fanden.

Der Gedanke, die Aneurysmen als Folge einer Thromboendarteritis 
metastatica purulenta, d. i. als embolische Aneurysmen aufzufassen, konnte in Anbetraeht der Tatsache, daß Patient an "Furunkulose" gelitten hatte, von vornherein nicht als gänzlich unwahrscheinlich erscheinen, mußte aber doch abgelehnt werden angesichts des Umstandes, daß an den Aneurysmen selbst alle Pathognomonika, die die Thromboendarteriitis metastatica purulenta auszeichnen, fehlten: so fehlte die eitrige Erweichung und die mehr oder minder kontinuierliche Ausbreitung, die embolische Aneurysmen charakterisieren. Schließlich sprach gegen den Gedanken, die Aneurysmen als Folge einer Thromboendarteriitis metastatica purulenta aufzufassen, auch die Tatsache, daß die Infarkte, die sich als Folgen der vielfach im Zusammenhang mit Aneurysmen eingetretenen Thrombosen fanden, typische "blande Infarkte" waren.

Eine tuberkulöse Basis den Aneurysmen zu substituieren, erschien sinnlos, angesichts der Tatsache, daß nirgends, weder in der Anamnese, noch im Krankheitsbericht, noch im Sektionsprotokoll von einer Tuberkulose die Rede war, sowie angesichts der Tatsache, daß speziell in den Aneurysmen selbst keine für eine tuberkulöse Infektion charakteristischen Befunde, keine Tuberkelbazillen, keine Tuberkel, keine Riesenzellen gesehen wurden.

Was die Annahme einer syphilitischen Genese betraf, so sprach weniges dafür, vieles dagegen; eine syphilitische Ätiologie der Aneurysmen konnte naheliegend erscheinen angesichts des Umstandes, daß der Patient'an Syphilis gelitten hatte und an ihr nach klinischer Angabe zugrunde gegangen war. Aber eine syphilitische Genese mußte sehr unwahrscheinlich erseheinen bei der völligen Integrität der Hirnarterien, die doch eine Prädilektionsstelle der Arteriitis syphilitica bilden, und mußte deshalb durchaus abgelehnt werden, weil die für die Arteriitis syphilitica charakteristischen histologischen Kriterien im histologischen Bilde der Aneurysmen völlig fehlten: nirgends eine Proliferation oder auch nur eine lamellöse Aufsplitterung der Elastica interna innerhalb der Wucherungen der Intima, nirgends Riesenzellen, nirgends Gummibildungen.

So mußte per exclusionem die Diagnose auf Aneurysmen einer Periarteriitis nodosa gestellt werden, eine Diagnose, die nichts gegen sich und alles für sich hatte; denn wasdie Periarteriitis nodosa auszeichnet, war vorhanden: im. makroskopischen Bilde vor allem die Beteilignng der Arterien des Herzens und der Niere, der Leber und des Darmes, im mikroskopischen Bilde vor allem die schmale seharfe Umgrenzung der GefäB-Rupturen; und was der Periarteriitis nodosa fehlt, fehlte auch hier, Granulombildung. Wueherung der Elastica interna und Beteiligung der ganzen Zirkumferenz der Gefäße.

So lautete schließlich die pathologisch-anatomische Diagnose: Periarteriitis nodosa obsoleta der Arterien des Herzens, der Nieren. der Leber und des Darms; kleinste Schwielenbildungen des Herzens: anämische Infarkte in der Leber und in beiden Nieren; ausgedehnte 
Infarzierung der Darmwand mit Nekrose und Geschwürsbildung der Darmschleimhaut; Hypertrophie der linken Herzammer.

Da aber die Diagnose auf- "Periarteriitis nodosa obsoleta" gestellt worden war, so mußte anch versucht werden, den Zeitpunkt zu bestimmen, wenigstens ungefähr zu umgrenzen, zu dem der nunmehr obsolete Prozeß akut gewesen war. Dies konnte nur auf Grund eines Vergleichs des hier vorliegenden Falles und der bisher bekannten Fälle geschehen. Das Studium der Literatur tührte aber zu folgenden Erwägungen:

1. Alle Fälle von Periarteriitis nodosa, die bisher beschrieben sind, trugen bei der Autopsie noch die Kennzeichen eines fortschreitenden Prozesses, d. h. der vorliegende Fall ist der erste Fall ron Periarteriitis nodosa obsoleta, der zur Beobachtung und Beschreibung gelangt.

Allerdings sind von KuBmaul-Maier, Pertik-Benedikt, Schmorl Fälle von Periarteriitis nodosa, die zur Genesung gelangten, also Fälle von Periarteriitis nodosa obsoleta beschrieben worden. Aber der Fall $\mathrm{KuBmaul-Maier} \mathrm{und} \mathrm{der} \mathrm{Fall} \mathrm{Benedikt-}$ Pertik sind nur klinisch beobachtet worden, nicht zur pathologischen Untersuchung gekonmen, und von dem Fall von Schmorl liegen wohl Daten der Klinik und der Autopsie, nicht aber Ergebnisse der histologisehen Untersuchung vor.

Und die Fälle Eppinger-Kundrat und Eppinger-Rokitansky, die übrigens klinischer Bemerkungen gänzlịch entbehren, verzeichnen, auch wenn sie im wesentlichen durch die Päthognomonika der "Periarteriitis nodosa obsoleta" charakterisiert sind, doch noch die verschiedenen Kriterien fortschreitender Entzündung, z. B. "sarkomähnliche Zellherde im adventitiellen und periadventitiellen Bindegewebe" usw.

2. Alle bisher beschriebenen Fälle von Periarteriitis nodosa haben sich dureh einen chronischen Verlauf ausgezeichnet, haben bis zu anderthalb Jahren gedauert, ohne daß im Leichenbefund noch allenthalben dentliche Zeichen eines fortschreitenden Prozesses gefehlt hätten. Wenn also nachgewiesen ist, daß die Periarteriitis nodosa anderthalb Jahre hindurch die Spuren des akuten Prozesses trägt, so muß ein Fall von Periarteriitis nodosa, der, wie der vorliegende Spuren von akuter Erkrankung nicht mehr aufweist, mindestens anderthalb Jahre alt sein.

Wenn aber einerseits dieser Fall vor mindestens anderthalb Jahren, also zu einer Zeit, da sich der Patient abgesehen von einer ganz peripheren Furunkulose noch vollkommen wohl befand, eingesetzt hat, wenn andererseits während der letzten anderthalb Jahre, als sich Patient in Behandlung befand, biș zum Tode des Patienten, nicht ein einziges Symptom auftrat, das den Ärzten auffallend, unerklärlich, außerhalb des Symptomenkomplexes der dem Patienten adhärenten Lres stehend erschien, so bedeutet dies, daB der vorliegende Fall für die Literatur der erste Fall von Periarteriitis nodosa ist, der vom Patienten unbemerkt, von den Arzten ungeahnt verlanfen ist, der erste Fall von Periarteriitis nodosa latens 
Allerdings wies auch der vorliegende Fall Spuren aknter Erkrankung auf. aber durchaus nicht mehr der Periarteriitis nodosa selbst, sondern nur in Form von Erkrankungen, die im Gefolge der Periarteriitis nodosa entstanden sind: Thrombosen und Folgezustände der Thrombosen, d. h. Organ-Nekrosen. Es hindert aber nichts anzunehmen, daß sich derartige Folgeerscheinungen erst nach Ablanf der Periarteriitis nodosa selbst ausgebildet haben; und es liegt aller Grund vor, zu vermuten, daß sich gerade in jüngster Zeit Thrombosen und deragemäß anch die durch die Thrombosen bedingten Organ-Veränderungen im Anschluß an die Salvarsan- resp. Quecksilberkur in der Nachbarschaft periarteriitischer Narben entwickelt haben, da einerseits festgestellt ist, daß Șalvarsan und noch melr Quecksilber durch Zerstörung von Blutkörperchen zur Entstehung von Thromben Yeranlassung geben, andererseits bekannt ist, dab Gefäßwandnarben Prädilektionsstellen für das Zustandekommen von Thromben bilden; und da schließlich gerade im vorliegenden Fall die Symptome der Thrombosenfolgen, speziell die Symptome der "Golitis necroticans ex thrombosi" sich im AnschluB an die Quecksilbertherapie gezeigt haben.

Im ganzen müssen wir demnach sagen: der vorliegende Fall bleibt für den Kliniker der erste Fall einer Periarteriitis nodosa latens, für den Pathologen der erste Fall einer Periarteriitis nodosa obsoleta.

II.

Wenn aber, wie wir einleitend hervorhoben, jeder Autor, dem wir eine Veröffentlichung eines Falles von Periarteriitis nodosa verdanken, ausgesprochen hat, daß vorläufig jeder Fall dieser Erkrankung als geeignet angesehen werden kann, einen Funken von Licht in das Dunkel zu bringen, das noch immer über dem Problem der Auslösung und des Ablaufs der P. n. (Periarteriitis nodosa) liegt, so sei auch im folgenden versucht, einerseits auf Grund exakter Durchsicht der Literatur, andererseits auf Grund der Beobachtung eigenen Materials za einer Deutung des Wesens der P. $\mathbf{n}$. zu gelangen. Ein solcher Versuch mag um so lohnender erscheinen, als die Antoren, die das Problem des Wesens der P. n. bisher in Angriff nahmen, im allgemeinen von isolierten Ergebnissen eigener Beobachtung ausgingen und demgemäß von den versehiedenen Autoren auch recht verschiedene Beobachtungen und Meinungen niedergelegt worden sind.

So liegen speziell in der Klinik der P. $n$. versehiedenartigste Angaben vor, Angaben, die vielfach keine Beziehung zueinander haben, geschweige denn als Grundlagen diagnostischer Überlegungen dienen können.

Wer die Krankengeschichten der P. n. miteinander vergleicht, der wird zunächst finden, daß darin allerdings einige Symptome mit großer Rege!mäBigkeit wiederkehren. So vor allem die Leukozytose und der. Milztumor, ferner der "chlorotische Marasmus" ( $\mathrm{KuBmaul-Maier)} \mathrm{und} \mathrm{die} \mathrm{große}$ Schwäche des Patienten. Aber diese Symptome sind nicht als speziell für P. n. pathognomoniseh zu verwerten: sie werden niemals den Kliniker auf die Diagnose der P. n. hinweisen können, sie werden höchstens dazu führen können, anzunehmen, daß eine akute Infektionskrankheit vorliege. Das gleiche 
gilt auch von den vagen Symptomen der Nephritis, wie Albuminurie und Hämaturie, ferner von den Symptomen der Herzschwäche wie Tachykardie, Blutdrucksenkung usw., die ziemlich regelmäßig in den Krankengesehichten der P. n. wiederkehren.

Jene Symptome aber, die nicht durchweg oder in der Mehrzahl der Fälle, sondern nur in den Beobachtungen einzelner Autoren die P. n. begleitet haben, lassen sich deshalb nicht für die Diagnose verwerten, weil sie, wie eine exakte Durchsicht der Krankengeschichten und ein kritischer Vergleich von Krankengeschichten und Sektionsprotokollen zeigen, nicht sowohl dureh die periarteriitischen Veränderungen selbst, als dureh die Folgeerscheinungen der P. n., durch die von der Arterienerkrankung gesetzten Organveränderungen, zu erklären sind: so z. B. die rheumatisehen Beschwerden durch Myositiden, die sich bei Obturationen von Muskelarterien entwickeln, so die Anurie, vielleicht auch die Albuminurie und jedenfalls die Ödeme dureh NierenInfarzierung nach Obturationen der Nierenarterien, so kolitische Symptome durch Nekrosen der Darmschleimhant nach Versehluß der Arteriae mesarrhaicae und die hemiplegisehen Symptome durch Aneurysmen der Gehirnarterien u. a. m.

Schließlich lassen sich noch einzelne Fälle von P. n. bezeichnen, die dadurch ausgezeichnet sind, daß sie überhaupt keinen "selbständigen" Symptomenkomplex aufweisen, sondern gleichsam unter der Symptomatologie einer anderen, ich möchte sagen "banalen" Infektionskrankheit verschwanden. So z. B. der Fall von Krzyszkowski, wo die Diagnose "Scarlatina" nahelag. ebenso die Fälle v. Bomhard-Oberndorfer und Hart-Künne; ferner der Fall Beitzke, der einer Streptokokkensepsis glich, u. a. m.

Derartige Fälle lassen sich für die Formulierung einer Klinik der P. n. natürlich erst recht nicht verwenden.

Aus all dem ergibt sich aber ein weiterer Schlub: wenn die Symptome der P. n. einerseits nur als Ausdrucksform einer Infektionskrankheit, vielleichteiner anderen Infektionskrankheit aufufassen sind, andererseits nichtaus den periarteriitischen Veränderungen selbst, sondern aus Veränderungen der von derP. n. betroffenen Organe abzuleiten sind, sokann zunäehst für die P. n. kein einheitlicher Symptomenkomplex aufgestellt werden, so kann fernerhin auch ein jeweils vorliegender Symptomenkomplex für die Deutung des Wesens der P.n. nicht herangezogen werden.

Deswegen sei nur in größter Kürze auf die Sonderstellung hingewiesen, die der vorliegende Fall innerhalb des klinischen Bildes der P. n. einnimmt. Er ist, wie schon gesagt wurde, der erste Fall von P. n., der außerhalb des Bewußtseins des Patienten selbst und anßerhalb ärztlicher Beobachtung abgelaufen ist. woraus, wie mir scheint, für die Klinik der P. n. wiederum die 
Folgerung resultiert, daß die P. n., die bisher in neunzig Prozent aller Manifestationen tödlich verlaufen ist, nicht nur zu günstigem Ausgang gelangen (KuBmaul-Maier, Sehmorl und Benedikt), sondern überhaupt latent ablaufen kann.

Immerhin hat die Erkenntnis, daß der Symptomenkomplex der P. n auBerordentliche Differenzen aufweist, wohl doch schon die Autoren dazu geführt, die Deutung der pathologisch-anatomischen Bilder für die Erklärung der Pathogenese der P. n. in erste Linie zu stellen.

Dies lag wohl zum Teil anch daran, daß fast alle Autoren, die Fälle von P. n. histologisch untersuchen konnten, Bilder beibrachten, die mit reichlicher Regelmäßigkeit einen außerordentlich charakteristischen Befund darstellten. Fast alle Autoren beschrieben im wesentlichen gleichartige Bilder: zunächst. im „ersten Stadium“, eine Nekrose der Media, verbunden mit einer Infiltration der Adventitia und zumeist auch einer Proliferation der Intima; im „Sekundärstadium“ eine Zunahme der Infiltration der Adventitia, eine beginnende Infiltration und eine zum Abschluß gelangende Nekrose der Media, verbunden mit einer gesteigerten Proliferation der Intima, und zwar durchweg mit dem Bemerken, daß sowohl die Infiltration der änßersten Gefäßwandschicht als die Proliferation der innersten Gefäßwandschicht in charakteristischer Weise gegen die mittleren Partien der Gefäßwand, die Media, gerichtet sind, im "dritten Stadium" ferner die Ausbildung eines indifferenten Narbengewebes an Stelle differenzierter Gefäßwand, und im Endstadium schließlich Bildungen von Thrombosen oder Aneurysmen.

Allerdings variierte die Deutung der Befunde darin, daß einzelne Autoren im Anfangsstadium nur eine Infiltration der Adventitia und erst späterhin eine Nekrose der Media sahen, andere Autoren zunächst eine Nekrose der Media und erst im Anschlub daran eine Infiltration der Adventitia bemerkten und wieder andere Forscher sogar eine Proliferation der Intima als Initialveränderung bezeichnen zu können glaubten. Dabei konstatiert man aber, was immerhin bemerkenswert erscheint, daß gerade die älteren Autoren zunächst in der Adventitia Veränderungen auftreten sahen ( $\mathrm{KuBm}$ a ul-Ma i e r, Zimmermann, Lorenz, Graf, Rosenblath, Freund, P. Müller. Vesprémi-Jankso, in nenerer Zeit Vera B lo ch), hingegen die neueren Autoren zunächst Störungen innerhalb der Media beobachten konnten (F errari, Mönckeberg, Dickson, Oberndorfer-v. Bomhard, Pertik, Hart-Künne, Benda, Beitzke), während schließlich eine ganz kleine Gruppe, die aber in nevester Zeit überhaupt keinen Vertreter mehr gefunden hat, sich auf den Standpunkt stellte, daß Veränderungen der Intima den ProzeB der P. n. einleiten (Weichselbaum-Chvostek, Fletcherv. Kahlden, Krzyskowski, Datnowski), and einzelne Forscher auch zu Kompromißvorschlägen gelangten (Schreiber, Versé, J. E. Schmidt. Guldner, G. B. Gruber). 
Bezüglich der Einzelheiten der Literatur sei auf die vor kurzem im „Zentralblatt für Herz- und Gefäßkrankheiten" erschienene Arbeit von G. B. Gruber verwiesen, die eine vorzügliche Zusammenfassung der bisherigen Beobachtungen enthält.

Derartige Versehiedenheiten in der Beurteilung der Initialveränderungen der P. n. führten dazu, daß fast jeder Autor, der einen Fall beobachten konnte, zu einer eigenen und eigenartigen Deutung des Ablaufs der Erkrankung gelangen konnte.

Kußmaul und Maier, die überhaupt als erste im Jahre 1866 die P. n. beschrieben haben, haben auch zuerst eine Erklärung ihrer Pathogenese zu geben versucht.

$\mathrm{KuBmaul}$ und Maier sahen als erste isolierte, pathognomonische Veränderung innerhalb einer im übrigen intakten Gefäßwand eine Leukozyteninfiltration in der Adventitia auftreten, sahen die Infiltration sich von außen nach innen gegen die Media vorsehieben. sahen danach die Media zugrunde gehen und an die Stelle zerstörter Media einerseits die von der Adventitia ausgehende Granulation, andererseits eine von der Intima aus erfolgende Bindegewebs-Proliferation treten, sahen derart schließlich an Stelle differenzierter und wertvoller Gefäßwand eine indifferente und wertlose Narbe treten. KuBmanl und Maier standen - wie dies der Zeit, in der sie lebten und wirkten, entsprach - auf dem Boden der Auffassung, daß eine progressive Metamorphose, wie eine Infiltration oder Proliferation, Initialveränderung einer Entzündıng sein könne. Danach gelangten sie zu dem SchlnB, daß der Prozeß der P. n. als EntzündungsprozeB von der Adventitia her die Gefäßwand ergreife, die Media zur Atrophie bringe, eine reaktive Proliferation der Intima hervorrufe und schließlich zur Entwicklung zirkumskripter Gefäßrupturen führe.

Dieser Betrachtungsweise schlossen sich später auf Grund gleichartiger Beobachtungen und gleichartiger Betrachtungen Zimmermann (1874), Rosenblath (1897), P. Mülle: (1899), Veszprémi-Jankso (1903), Vera Bloch (1913) an; in gewissem Sinne auch Graf (1897) and Freund (1899; die annahmen, dab der ProzeB der P. n. speziell von den innerhalb der Adventitia gelegenen Partien der Vasa vasorum aus einsetze.

Aber ehe noch diese Autoren alle Gelegenheit hatten, sich auf Grund eigener Beobachtung zur Frage der P. n. zu äußern, wurde eine nene Auffassung ihres Wesens von Chvostek and Weichselbaum aufgestellt.

Chvostek und Weichselbaum untersuchten im Jahre 1878 einen Fall, der außer den Zeichen akuter Erkrankung, und zwar akuter Entzündung, schon Endstadien des Prozesses in Form von Aneurysmen-Bildungen aufwies. Sie fanden, daß in den Zentren solcher Herde eine gleichmäßigge Zerstörung aller drei Gefäßwandschichten den irreparablen Abschluß eines abgelanfenen Prozesses erkennen ließ, daß ụm die Zentren Infiltration der Adventitia, Nekrose der Media und Proliferation der Intima den Höhepunkt eines fortschreitenden Prozesses darstellten, daß aber an den Peripherien solcher Herde stets nur eine Proliferation der Intima zu sehen war. Sie zogen daraus den Schluß, daß die Peripherie der Herde den Beginn des Prozesses erkennen lasse, und nahmen daher an. daß eine Proliferation der Intima den Anfang des Prozesses der P. n. darstelle, dab erst als sekundäre und reaktive Erscheinungen danach eine Infiltration der Adventitia einsetze, und schließlich aus der Vereinigung der von der Adventitia hersprossenden Granulationen und der von der Intima her vorgetriebeneu Proliferationen die Zerstörung: der Media resultiere, durch deren Ausfall die Bedingungen für die Entstehung der Gefäßwand-Rupturen gegeben seien.

Ihrer Auffassung folgten später Fletcher (1892) und v. Kahlden (1894), Krzyszkowski. (1899) und Datnowski (1908). So entstanden kurz nach der Entdeckung der P. n. und kurz nacheinander zwei Theorien, von denen die eine das Initialstadium der Erkrankung in die außerste, die andere in die innerste Gefüßwandschicht verlegte; so 
entstand später auch ein Kompromiß-Vorschlag, in dem Schreiber (1904) den Gedanken zur Erwägung stellte, alle Gefäßwandschichten als Basis der P. n. zu betrachten.

Hingegen war nie von dem Gedanken die Rede, den Ausgangspunkt des Entzündungsprozesses, den die P. n. darstelle, in der mittleren Gefäßwandschicht, in der Media zu suchen, schlechthin deshalb nicht, weil alle Autoren, wenn sie wirklich Initialstadien der Erkrankung vor Augen hatten, nur eine Infiltration der Adventitia oder in seltensten, und wie späterhin festgestellt wurde, zumeist zweifelhaftesten Fällen eine Proliferation der Intima saben. Daher kam es auch, daB der erste, der den Ursprung der P. n. in die Yedia verlegt wissen wollte, Mönckeberg, nicht auf Grund effektiver Beobachtungen. sondern auf Grund theoretischer Überlegungen ein derartiges Postulat erhob.

Mönckeberg komte de facto im wesentlichen die gleichen Beobachtungen über die Anfänge der P. $\mathrm{n}$. beibringen, wie alle, die vor ihm Anfangsstadien beschrieben hatten: anscheinend gleichzeitig stellt sich eine Nekrose der Media und eine gegen die Media hin gerichtete Infiltration der Adventitia ein, anmittelbar im Anschluß daran eine Wucherung der Intima, die ebenfalls gegen die Media gerichtet ist. Aus der Vereinigung der von der Adventitia her sprossenden Granulationen und der sich von der Intima her vorschiebenden Proliferationen entsteht, bei völligem Zugrundegehen der Media, eine zirkumskripte Gefäßwandnarbe, die weiterhin zur Basis von Aneurysmen und Gefäßwandrupturen werder oder zu Thrombosen und GefäBobturationen führen kann.

Mönckeberg brachte zar Klärung des Wesens der P. n. des weiteren theoretische Überlegungen bei: er ging von der Ubberlegung aus, .daß die Angaben aller Autoren in einem Punkte übereinstimmen: stets wurde ein Einwuchern anfangs von Rundzellen, später von Granulationsgewebe in die Media von der Umgebung aus beobachtet. Vergegenwärtigen wir uns, daß sowohl die Lenkozyten als auch die nicht seßhaften Zellen jungen Bindegewebes mitsamt den Kapillarsprossen in ihrer Wander- resp. Wachstumsrichtung chemotaktischen Einflüsen unterworfen sind, so kommen wir bei der Betrachtung obiger stets erhobener Befunde mit unzweideutiger Sicherheit zu dem Schltusse, daß nar zwischen Intima und Adventitia, mithin in der Media der Sitz des chemotaktischen Reizes lieger kann. Da dieser aber von Anfang an vorhanden zu sein scheint, werden wir nicht fehlgehen, wemn wir auch die primäre Gewebsläsion in die Media verlegen. Durch das Einwuchern der Zellen wird nicht, wie einige Autoren es darstellen, die Media zerstört. sondern die Zellen wuchern in die Media ein, weil diese bereits primär geschädigt ist unở die Zerfallprodukte jene Elemente chemotaktisch beeinflussen". So kam Mönckeberg zu der Forderung, die Media als den Ausgangspmnkt der P. n. zu betrachten.

v. Bomhard-Oberndorfer (1907, 1908), Beitzke (1908), Hart-Künne (1909, 1910), Dickson (1907), Muir (1907) schlossen sich dieser Forderung, die Media als den Ort der primären Gewebsalteration bei der P. n. anzusehen, an, aber nicht mehr auf Grund theoretischer Uberlegungen, sondem auf Grund tatsächlicher Beobachtungen: weil sie in ihren Fällen eine Degeneration der Media vor einer Infiltration der Adventitia oder gar einer Proliferation der Intima sahen.

Immerhin wurden ungefähr zur selben Zeit, da Mönckeberg die Media als den Ausgangspunkt der P. $n$. bezeichnete, von Versé Untersuchungen veröffentlicht, auf Grunở deren der Autor zu einer anderen Ansicht gelangte.

Versé konnte nämlich in Untersuchungen mehrerer Fälle von P. n. im allgemeinen Bilder beobachten, in denen als erste Veränderung der P. $n$. eine an der Genze von Adventitia und Media entstehende Exsudation auftrat, weiterhin Bilder, in denen sich die P. n. zuerst in Form einer leichten, fleckförmig begrenzten Infiltration der Adventitia äußerte, oder anch Bilder, die man in der Richtung einer primären Nekrose der Media deuten konnte. Da Versé auf dem Standpankt steht, daß sowohl eine regressive Metamorphose, wie eine Nekrose, als auch eine progressive Metamorphose, wie eine Infiltration oder auch Erscheinungen seitens des Gefäßapparats, wie eine Exsudation als gleichwertige

Virchows Archiv f. pathol. Anat. Bd. 22\%. Heft 1. 
Erstlings-Manifestationen einer Entzündung gelten köunten, so nahm Versé an, daß der Prozeß der P. n. sowohl in der Adventitia als auch in der Media einsetzen kann, im allgemeinen aber Adventitia und Media speziell in ihrem Grenzgehiet gleichzeitig befällt und ,von außen nach innen“" fortschreitet.

Neben all diesen Gedankengängen aber, die, wenn sie auch im einzelnen weitgehende Differenzen zeigten, doch sämtlich darin übereinstimmten, daß sie in der P. n. einen EntzündungsprozeB sahen, wurden noch weitere Ansichten ausgesprochen, die die P. n. überhaupt nicht als 'Entzündungsvorgang ansehen wollten, sonderm gleichsam als den Ausdruck einer Störung mechanischer Korrelationen.

Der Erste, der mit einer solchen Ansicht vor die Offentlichkeit trat, war P. Meyer (1878). Er ging von der Beobachtung eines Falles aus, in dem die Spätstadien der Erkrankung und darunter wiederum speziell die Aneurysmen and Thrombosèn dominierten, wenn sich auch noch Bilder erkennen lieBen, die durchaus als Manifestationen eines fortschreitenden Prozesses angesehen werden konnten, z. B. Infiltration der Adventitia, Nekrose der Media, Proliferation der Intima. P. Meyer beschrieb demnach im wesentichen die gleichen Bilder, die allgemein als charakteristische Ausdrucksformen der P. n. angesehen werden, gelangte aber bei dem Versuch, die Beobachtung der isolierten Erscheinung des Einzelfalles zu einer Synthese des Gesamtbildes der Erkrankung zu exheben, zu ganz eigenartigen Ergebnissen:

„Sowohl in den größeren Knoten der Arterien der Eingeweide und der Gliedmaßen. wie in den kleinen, fast mikroskopischen Verdickungen der Arterien-Astchen haben wir Tomente gefunden, die ganz zweifellos aut eine Ruptux der Gefäßwand deuten. Gegenüher dem allen diesen Herden gemeinsamen Moment erseheinen die übrigen Veränderungen, die thrombotischen Abscheidungen, die hyalinen Massen, wie endlich die Infiltration der adventitiellen GefäBschichten, ja sogar die Erweiterung als unbeständig und sind schon deswegen als sekundäre Folgen der Rupturen anzusehen. Wie ersichtlich, sind von der Ruptur hauptsächlich jene Bestandteile der Arterienwand getroffen, welche dem Gefäß eine Resistenz verleihen, die eigentliche Muskelhaut und jene elastischen Fasern, welche entweder in dex ganzen Dicke der Media oder um diese herum in den innersten Schichten der Adventitia das Stützwerk der Wandung bilden." P. Meyer schlob dann des weiteren, daß eine Ruptur Aer mittleren Gefäßwandschicht nicht nur das Charakteristikum, sondern auch das primäre Moment der P. n. sei, und kam, da einer derartigen Ubberlegung die Klärung der Ätiologie sehr nahe lag, zu dem Schluß, daß abnorme Blutdrucksteigerungen infolge eines ausschweifenden Lebenswandels die Ursache der P. n. seien,

Auf P. Meyer folgte Eppinger (1887).

Auch Eppinger beobachtete zwei Fälle, die durch die Ausbildung von Spätstadien, anch speziell von Aneurysmen charakterisiert waren. Und er kam ebenfalls zu der Uberzeugung, daß Störungen eines mechanischen Gleichgewichts, das im Gefäßsystem zwischen Blutdruck einerseits und Resistenz der Gefäßwand andererseits bestände, die P. n. ausIösen. Er gelangte dazu allerdings.zunächst, indem er die Annahme, nach der die P. n. ein syphilitischer Prozeß sei, ausschloß. Dies versuchte er durchzuführen, indem er betonte, dah einerseits die für die P. n. charakteristischen Erscheinungsformen, die Aneurysmen, bei syphilitischen Prozessen nie zu beobachten seien, andererseits die für die Syphilis charakteristischen Gummibildungen und Nekxosen im Bỉlde der P.,n. fehlten: .Wenn man aber jemals einem Prozeß eine gewisse, fast starre Konsequenz seiner anatomisch-histologischen Verlaufsweise nachrühmen kann, so ist es die Syphilis." Eppinger versuchte sodann überhaupt eine entzündliche Ätiologie der P. n. auszuschalten. Er glaubte dies tun zu können, wenn er danlegte, daß es sich weder um eine "Endarteritis chronica“, alias Atherosklerose, noch um eine vom Parenchym benachbarter Organe aus übergreifende Entzündung, noch etwa um embolische Aneurysmen handeln könne. Ferner auf Grund der Überlegung, daß - wenn anch angenommen werden müsse, daß die Ein- 
gänge der Aneurysmen durch fortgesetzte Wucherung der Intima am Halse der schon bestehenden Aneurysmen eingeschränkt sind, so sind die Eingänge der Aneurysmen doch noch viel zu klein, um einem Entzïndangsherd" zu entsprechen", schließlich auf Grund der Überlegung, - „daß diese Sorten von Aneurysmen außerordentlich selten, arterielle Entzindungen aber bedeuteter Charaktere eben sehr oft, und zwar ohne Aneurysma-Bildung, vorkommen".

Des weiteren glaubte Eppinger, die „Ausbuchtung der Artexien, in deren Grenzen die Elastika abgesetzt oder abgerissen erscheint, in deren Wandung die Intima gleichmäßig und ohne Veränderung, die Muskularis aber in sich verjüngendem Zustande sich fortsetzt, wobei die Verjüngung so fortschreitet, daß den Kuppelabschnitten die Muskalaris vollständig fehlen kann, als Aneurysmen in ihrem ersten Entwicklungsstadium ansehen zu müssen". Ex glaubte danach die Entwicklung der P. n. mit einer isolierten Ruptur der Elastica interna beginnen lassen zu können und glaubte, daß, ,da die Intervention eines syphilitischen oder eines entzündlichen oder eines ähnlich gearteten destruierenden Prozesses ausgeschaltet werden könne, gar nichts anderes übrig bleibe, als an eine für sich auftretende, ganz und gar von benachbarten und andrängenden materiellen Störungen unabhängige Unterbrechung oder Ruptur der Elastika zu denken".

So nahm er eine spontane Ruptur der Elastika als den Beginn der P. n. an, wie hier schon vorweggenommen sei, auf Grund einer kongenitalen Debilität der Elastika.

Die Ansehauung Eppingers fand keine weiteren Anhänger; ebensowenig eine Ansicht, die erst in neuester Zeit ausgesprochen wurde, die aber innerhalb der Entwicklung der Erforschung der P. n. überhaupt isoliert steht, die Ansicht von Ferrari.

Fexrari beobachtete 1903 einen Fall und glaubte nach dem histologischen Befund als erstes Stadium der P. n. einerseits eine Erweiterung dex Gefäßquersehnitte, andererseits das Auftreten eines serösen Odems zwischen auseinander gedrängten Fasern der Media betrachten zu dürfen, woran sich exst als sekundüre Reaktionen Degenerationen der Yedia und reaktive Veränderungen der Adventitia resp. Intima anschlössen. Er nabm an, daß eine Lähmung der Gefäßwandmuskulatur das erste Stadium der P. n. hervorrufe, und gelangte im Anschluß daran zu der Auffassung, daß eine durch toxische Agentien, speziell die Wirkung des Alkohols bedingte Lähmmng der Gefäßnerven-Zentren eine Degeneration der Gefäßwandmuskulatur als Beginn der P. n.auslöse, - eine Anschaunng, die viel Widerspruch aber keine Gefolgschaft fand. -

III.

Der Fall, der mir selbst vorgelegen hat, ermöglicht es allerdings nicht, an Hand effektiver Beobachtungen ein Urteil bezüglich der Pathologie der Anfangsstadien der P. n. abzugeben, schlechthin deshalb nicht, weil es sich um einen Fall von Periarteriitis handelt, der nur noch das Endstadium der P. n. zeigt, irgendwelcher akuter Veränderungen aber vollkommen entbehrt.

Wemn ich selbst im folgenden versuchen möchte, zu einer Schlußfolgerung bez. der Pathogenese der P. n. zu gelangen, so fühle ich mich daher gezwungen an der Hand einer kritischen Durehsicht der bestehenden Anschanungen, eines Studiums vorhandener Beobachtungen vorzugehen; und dies mag vielleicht nicht zwecklos erscheinen angesichts der Tatsache, daß, wie oben bereits betont, die Autoren, die bisher über P. n. gearbeitet haben, zumeist geneigt waren, eigene Beobachtungen $\mathrm{zu}$ wesentlichen Grundlagen 
ihrer Auffassung zu machen und daraufhin zu. jeweils recht verschiedenen. reichlich subjektiven Anschauungen vom Wesen der P. n. gelangten.

Es bestehen, um dies noch einmal in Kürze zu rekapitulieren, bis jetzt acht Auffassungen über das Wesen der P. n.:

1. Die von Kußmaul und Maier, Infiltrationen der Adventitia als primärer Ausdruck eines Entzündungsprozesses.

2. Die von Chvostek und Weichselbaum, Proliferation der Intina als primäre Manifestation eines Entzündungsvorganges.

3. Die von Mönckeberg, Nekrose der Media als Beginn der P. n. im Sinne der primären Gewebsalteration eines Entzündungsprozesses.

4. Die von Schreiber, primäres Einsetzen eines Entzündungsprozesses in allen drei Gefäßwandschichten.

5. Die von Versé, primäres Einsetzen einer entzündlichen Kreislantstörung im Grenzgebiete von Adventitia und Media.

6. Die von P. Meyer, primäres Einreißen der mittleren Gefäßwandschicht als Ausdruck einer regressiven Metamorphose, die durch Störung eines mechanischen Gleichgewichts, speziell durch abnorme Blutdrucksteigerung bedingt wird.

7. Die von Eppinger, primäre Ruptur der Elastika, ebenfalls als Ausdruck einer rein regressiven Metamorphose, die, ätiologisch gesprochen, durch eine kongenitale Debilität der Elastika bedingt wird.

8. Die von Ferrari, primäre Degeneration der Media, als rein regressive Ketamorphose infolge Lähmung der Gefäßwandmuskulatur.

Zunächst scheint mir das Eine zweifellos festzustehen, daß es sich bei der P. n. um einen Entzündungsprozeß, sogar um einen „exquisit entzündlichen Prozeß" (Mönckeberg). handelt. Dies scheint mir mit absoluter Deutlichkeit sowohl aus den klinischen Angaben, die die einzelnen Fälle begleiten, als auch aus den anatomischen Befunden, die bis jetzt vorliegen, hervorzugehen.

Klinisch vor allem aus der Tatsache, dab in allen Fällen von P. n., in denen das Blutbild untersucht wurde, eine Leukozytose, zumeist sogar eine recht hochgradige Lenkozytose verzeichnet wurde; daB ferner in der Mehrzahl der Fälle, die über das Verhalten der Milz Aufschluß geben, eine Schwellung der Milz beobachtet wurde; vielleicht auch aus einer Wertung des Umstandes, daß nicht wenige Fälle von $P$. n. vorliegen, in denen während ihres Ablaufs eine akute Nephritis oder eine akute Endokarditis einsetzte, also Komplikationen, die wohl als Begleiterscheinungen von entzündlichen Allgemeinerkrankungen gelten dürfen.

Anatomisch wohl vor allem aus dem Umstand, daß von allen Autoren. die Anfangsstadicn der P. n. beobachten konnten, innerhalb derselben eine hochgradige Infiltration in Verbindung mit einer Nekrose gesehen wurde. Dazu ist aber zu bemerken: wohl kann eine Nekrose von Gewebsbestand- 
teilen und im Anschluß daran eine Infiltration der Gewebe auch bei nicht entzündlichen Prozessen gesehen werden; derartig intensive Nekrosen und derartig hochgradige Reaktionsvorgänge, wie die, die bei der P. n. von allen Autoren gesehen wurden, müssen doch, nach dem derzeitigen Stand unserer Kenntnisse, der Entzündung reserviert bleiben. Danach kann wohl gesagt werden, daß es sich bei der P. n. um einen Entzündungsprozeß handelt, und zwar um eine entzündliche Systemerkrankung der kleinen Arterien. Wenn aber die P. n. als EntzündungsprozeB angesehen wird, so müssen aus der Erklärung ihrer Genese von vornherein diejenigen Erklärungsversuche ausgeschaltet werden, die in der P.n. schlechthin eine regressive Metamorphose sehen; also die Ansicht von P. Meyer, der die P. n. als einen Komplex von Zerreißungen kleinerer Gefäße, bedingt durch hohe Blutdrucksteigerung infolge eines aússchweifenden Lebenswandels, ansah; ferner die Auffassung von Eppinger, der die P. n. mit einer Zerreißung der elastischen Lamellen infolge einer Schwäche der kongenitalen Anlage einsetzen lieb; schließlich auch die Auslegung von Ferrari, der eine Lähmung der Gefäßmuskulatur bezw. eine Lähmung der die Gefäßmuskulatur innervierenden Nerven als Initialstadium der P. n. betrachtete.

Im eimzelnen läßt sich gegen die Anschauung von P. Meyer noch anführen, daß es schlechthin unmöglich ist, durch ausschweifenden Lebenswandel bedingte Blutdrucksteigerungen als Ursache einer Erkrankung anzusehen, die bei Kindern von wenigen Monaten oder wenigen Jahren (cf. Fall Krzyszkowski und Fall Eppinger-Kundrat) beobachtet worden ist. Fernerhin läßt sich der Umstand geltend machen, daß überhaupt eine Gefäßruptur niemals durch den Blutdruck allein erzeugt werden kann. Gegen die Meinung ron Eppinger ist aber die Tatsache anzuführen, daß P. n. bei alten Frauen am Eingang des Senium aufgetreten ist (cf. Fletcher und Fall r. Kahlden), und daß es doch schwer fällt anzunehmen, daß eine kongenitale Mißbildung, als die eine angeborene Debilität der Elastika doch betrachtet werden muB, bis zum Eintritt des Senium latent bleiben kann. Auch die Überlegung, daß Veränderungen, die auf einer kongenitalen Debilität des Gefäßsystems beruhen, sich doch wohl im ganzen Verlauf des Gefäßsystems zeigen müssen, und nicht, wie bei der P. n. nur in regionärer Ausdehnung und nur in herdförmiger Begrenzung, - spricht gegen Eppinger. Gegen Ferrari muß aber der Umstand bewertet werden, daß eine durch Lähmung der Gefäßmuskulatur ausgelöste Erkrankung sich doch wohl an der Gesamtheit der Zirkumferenz der Gefäßwand und im ganzen Verlauf ron Gefäßen abbilden muß, während die P. $n$. doch immer nur in knötchenförmiger Beschränkung auftritt und nach Angabe aller Autoren gerade durch die schmale Begrenzung und scharfe Absetzung der Erkrankungsherde charakterisiert ist.

So bleiben zur Erklärung des Wesens der P. n. nur jene Auffassungen, 
die darin übereinstimmen, daß sie die Erkrankung als einen Entzündungsprozeß betrachtet wissen wollen und die nur darin differieren, daB sie entweder die Adventitia (Kußmanl-Maier), oder die Media (Mönckeberg) oder die Intima (Chvostek-Weichselbaum) oder die beiden äußeren Gefäßwandschịchten (Versé) oder gar alle drei Gefäßwandschichten (Schreiber) als primären Sitz der Erkrankung ansehen.

Jede Ansicht aber, die die P. n. als Entzündungsprozeß erklären will, muß auf dem Boden einer Auffassung des Wesens der Entzündung stehen, welche alle sogenannten Entzündungsvorgänge umschließt und kausal auseinander sich entwickeln läBt. Eine solche Auffassung ist aber m. E. nur in der namentlich von Lubarsch anf dem Boden der Weigertschen Lehre begründeten Eutzündungslehre zu sehen.

Nach Lubarsch verstehen wir unter Entzändung die Kombination von Gewebsalteration, Exsudation und Gewebsproliferation, wobei die Alteration als das primäre Moment anzusehen ist, von dem die übrigen Vorgänge entweder direkt oder indirekt kausal abhängen.

Wer sich aber heute anf den Boden dieser Theorie stellt, der wird das Folgende anerkennen müssen.

Speziell bei der P. n. kann weder die Infiltration der Adventitia, auch wenn sie "zuerst" gesehen wurde, noch die Proliferation der Intima, auch wenn sie "isoliert" auftrat, als primäre Manifestation der Entzündung angesprochen werden, sondern es mub betont werden, daB die Betrachtung der P. n. die Aufgabe stellt, nach Bildern zu suchen, in denen wirklich eine primäre Gewebsalteration und damit das Initialstadium des Entzündungsprozesses festgestellt werden kann. Derartige Alterations-Erscheinungen könnten a priori sowohl in der Adventitia als auch in der Media oder in der Intima vorhanden sein; es $m u ß$ aber in Betracht gezogen werden, daß sowohl die Reaktionserscheinungen der Adventitia, d. i. die Infiltration, als auch die Reaktionserseheinung der Intima, d. i. die Proliferation, gleichermaßen und in ausgesprochenster Weise gegen die Media zu gerichtet erscheinen, und es muß daraufhin vermatet werden, daß der Herd der primären Alteration und Sitz des chemotaktisch wirksamen, die Emigration und die Proliferation in ihrer Richtung beeinflussenden Reizes in der Media lokalisiert ist. Angesichts der Tatsache nun, daß fast sämtliche modeme Autoren, die doch zum mindesten über die besten Hilfsmittel der Techuik verfügten, in ihren Fällen als pathognomonische Veränderung zuerst eine isolierte Nekrose der Media sahen. muB zweifellos die Media als Sitz der primären Gewebsalteration angesprochen werden.

Wenn aber eingewandt wird, daß andere, ältere Autoren Bilder sahen, in denen eine Infiltration der Adventitia vior der Nekrose der Media einsetzte, so läßt sich dem erwidern: die Pathologie kennt zahlreiche Beispiele, in denen Vorgänge der Gewebsalteration substituiert werden, und zwar von 
allen Forschern vorausgesetzt werden, ohne daß es auch nur einem einzigen gelungen ist, sie tatsächlich sichtbar zu machen, z. B. zahlreiche Prozesse im Nervensystem; man ist daher berechtigt anzunehmen, daß in denjenigen Fällen von P. n., in denen von den Beobachtern bei den Anfangsstadien nur eine Infiltration der Adventitia oder gar eine Proliferation der Intima beobachtet wurde, die Alteration der Media wohl existierte, aber aus einer Unvollkommenheit der Hilfsmittel leraus nicht sichtbar gemacht werden konnte.

Daraus resultiert schließlich; daß jene Beobachter, die eine Nekrose der Media als erste Manifestation der P. n. sahen, wirklich das Primärstadium der P. n. beschrieben haben, alias, daß die P. n. mit einer Nekrose der Media einsetzt, an die sich eine Infiltration der Adventitia und eine Proliferation der Intima als Reaktionsmaßnahmen des Organismus anschließen.

Denn was für die Infiltration gilt, gilt natürlich auch für die Proliferation der Intima, auch sie kann nur als Selzundärerscheinung aufgefaßt werden; ganz abgesehen davon, daß die Antoren, die die Periarteriitis in der Intima einsetzen ließen, wohl Irrtümern anheimgefallen sind, die aber im einzelnen darzustellen, nur zu einer Wiederliolung älterer Ausführungen führen würde (vgl. Versé). -

Wenn ich gezwungen war, mich über die Pathogenese der Anfangsstadien der P. n. allein an der Hand der Angabe anderer Autoren zu orientieren, so möchte ich versuchen, bei der Beurteilung der Endstadien der P. n. nicht nur die Bilder, die von älteren Autoren gegeben sind, sondern auch eigene Beobachtungen zu verwerten.

Endstadien der P.n. sind wohl von allen Autoren beobachtet und von allen in gleicher Weise beschrieben worden. An Stelle der zugrunde gegangenen Gefäßwand entsteht eine aus solidem Bindegewebe bestehende Narbe, die als "heterotyper" Bestandteil des im übrigen gleichmäßigen Gefäßwandgewebes zu Thrombosen oder Aneurysmen Veranlassung gibt. Es ist auch von allen Autoren behauptet worden, daß das Narbengewebe einen völlig indifferenten Charakter trägt und selbst anch keinem weiteren Differenzierungsprozesse mehr verfalle, weder regressiven Metamorphosen wie Verkäsung oder Verkalkung, noch auch metaplastischen Prozessen wie etwa einer Neubildung elastischer Fasern.

Nun ist auch schon hier und da in Erwägung gestellt worden, daß̧ die Endstadien der P. n., die bisher beobachtet worden seien, vielleicht doch nicht alt genug seien, um die Frage nach der Fähigkeit der P. n., im Spätstadium regressive Metamorphosen oder metaplastische Umwandlungen durchzumachen, schlechthin zu verneinen.

Im speziellen hat Georg B. Gruber hierüber geschrieben: „doch glaube ich, daß bei längerer Dauer der Krankheit doch auch Regenerationsprozesse der Elastika einsetzen können, die in der Bildung von netzförmigen, aus 
Schleifen und Maschen bestehenden Systemen elastischer Fasern bestehen. Dies ist bisher nur von Abramow für seinen ersten Fall berichtet worden. Leider besitzen wir in dieser Hinsicht keine genaueren Angaben über die Befunde im Fall Schmorl. Natürlich können hier nur protrahierte oder geheilte Affektionen in Frage kommen. Es bleibt den künftigen Untersuchern vorbehalten, diesem Punkt, nämlich der Regeneration der Elastika, ihr Augenmerk zuzuwenden. Vorläufig möchte ich es fast ansehen, als sei für die P. n. eine gewaltsame Zerstörung des elastischen Gefäßgewebes ohne Regeneration recht charakteristisch." De facto handelt es sich bei dem "ersten Fall Abramow" um folgendes:

Abramow beschrieb im Jahre 1899 die Erkrankung eines jungen Mannes, die von ihm selbst als Arteriitis syphilitica aufgefaßt wurde, die aber im makroskopischen Befunde insofern einer P. n. glich, als sich nicht im Gehirn, wohl aber am Herzen Aneurysma-Bildungen fanden. Der mikroskopische Befund zeigte starke Infiltrationen und Granulationen der Adventitia, die gegen die Media vordrangen, Nekrosen der Media in ausgedehntestem Maße und Wucherung der Intima; diese war von einem mit der Elastica interna im innigsten Zusammenhang stehenden System elastischer Fasern durchsetzt, das geradezu als eine lamellöse Aufsplitterung der Elastika imponierte.

Auberdem beschrieb Abramow einen zweiten Fall von Arteriitis syphilitica, ebenfalls bei einem jungen Nann, der auch durch die Ausbildung aneurysmatischer Gefäßerweiterungen mit spez. Lokalisation an den Koronararterien charakterisiert war und folgenden mikroskopischen Befund hatte: Infiltration und Granulation der Adventitia, die auf die Media übergreift, Degeneration und Nekrose der Media, vereinzelt mit käsigem Zerfall, Wucherung der Intima mit massenhafter Durchsetzung elastischer Fasern, die sowohl als eine lamellöse Aufsplitterung der Elastika, wie auch als eine echte Neubildung elastischer Fasem gedeutet werden kann.

Beide Fälle wurden, wie gesagt, von Abramow als Arteritis syphilitica angesehen. Doch wurde späterhin betont (v. Kahlden, Versé), daß Abramow vielleicht über das Bild der P. n. nicht hinreichend instruiert gewesen sei. Darnach wurde der erste Fall von Abramow von v. Kahlden als ein Fall von P. n. bezeichnet, und zwar nur anf Grund der makroskopischen Lokalisation: er hat seither in der Literatur durchweg als Fall von $\mathrm{P}$. n. figuriert. Über die Rubrizierung des zweiten Falles von Abramow hat sich in der Literatur keine Ubereinstimmung der Autoren entwickeln können, da er von der Mehrzahl der Autoren nicht zur P. n. gerechnet wurde, hingegen von einzelnen Forschern, und zwar gerade Forschern, die sich größter Autorität auf dem Gebiete der Gefäßkrankheiten erfreuen, als der P. n. zugehörig angesprochen wurde (Benda, Versé), von einzelnen sogar als ein Bindeglied zwischen Arteriitis syphilitica und P. n. angesehen wurde (Versé). 
Ich glaube sagen zu dürfen, daB die Abramowsche Publikation, ob man nun die beiden Fälle als Fälle von P. n. auffassen will oder nicht, für die Frage nach der Fähigkeit der P. n., bei längerer Dauer Regenerationsprozesse der Elastika einsetzen zu lassen, irrelevant bleibt; und zwar deshalb, weil gerade die Fälle ron Abramow allzu deutliche Bilder einer akuten Erkrankung tragen, als däß es möglich wäre, sie zu irgendwelchen Schlüssen über die Prozesse des Spätstadiums der P. n. heranzuziehen. Ich kann daher, was im allgemeinen die Frage nach den Bildern der Spätstadien der P. n. anbelangt, resümieren, daß einerseits alle Autoren, wenn sie wirklich Endstadien der Erkrankung beschrieben, ein indifferentes Narbengewebe zeichneten: daß andererseits auch der vorliegende Fall, der, doch sicherlich einen sehr alten Fall darstellt, nirgends Bilder erkennen ließ, die etwas anderes waren als indifferente Endprodukte einer indifferenten Entzündung.

Im ganzen können wir also über die Pathologie der P. n. das Folgende sagen: Die Periarteritis nodosa ist ein entzündlicher ProzeB, und zwar eine entzündliche Systemerkrankung der kleinen Arterien. Siesetzt ein miteiner Nekroseder mittleren GefäBwandschicht, der Media, der rasch von beiden Seiten her, d. h. von der äuBeren und inneren GefäBwandschichtaus, Reaktionstorgänge folgen: die Adventitia wird durchsetzt von einem fibrinösen Exsudat, das sich zunächst sowohl in den inneren Partien der Adventitia; als auch in den äuBeren Schichten der Media lokalisiert, ferner von leukozytären Elementen, die gegen die Media vordringen, schlieblich von einem Granulationsgewebe, das, die Elastica externa durchbrechend. in die Media eindringt; gleichzeitig wandelt sich die Intima za einem starkproliferierenden Bindegewebe um, das e inerseits durch die Elastica interna in die Media eindringt, die inzwischen einer völligen Nekrose verfallen ist, andererseits in das Gefäßlumen vorwuchert. So setzt sich an Stelle spezifisch gebauter und spezifisch funktionierender Schichten differenter and resistenter Gefäßwand ein indifferentes und irresistentes $\mathrm{Narbengewebe.}$

IV.

Aus klinischen Darstellungen und pathologischen bezw. pathogenetischen Deatungen der P. n. muß, bei dem völligen Fehlen regelmäBiger, d. i. verwertbarer bakteriologischer Notizen, das Problem der Atiologie der P.n. in Angriff genommen werden.

Im allgemeinen haben fast alle Autoren von der Betrachtung der klinischen Symptome und der pathologisehen Ausdrucksform aus die Ätiologie 
der P. n. zu ergründen versucht. Dementsprechend sind, wie die klinischen Bilder differierten und wie über die Pathogenese die verschiedenartigsten Deutungen ausgesprochen wurden, auch bez. der Ätiologie der P. n. die mannigfachsten Anschaungen zustande gekommen.

So glaubten Kußmaul und Maier, die Lues als Ursache in Frwägung ziehen zu müssen, ebenso Chrostek und Weichselbaum, Graf, Schmorl. Versé, Paul Nüller. Andere Autoren (v. Kahlden. Fletcher, Rosenblath, Mönckeberg, Beitzke, J. E: Schmidt und unter schärtster Betopung Veszprémi-Jankso) glaubten einen unbekannten Erreger als ätioologisches Moment in Anspruch nehmen zu dürfen. Wieder andere wie Hart-Künne and G. B. Gruber sahen in der P. n. überhaupt nicht ein ätiologisch einheitliches Krankheitsbild, sondem den nur pathologisch einheitlich charakterisierten, ätiologiseh verschiedenartigen Erregern zuzusprechenden Folgezustand differenter Infektionskrankheiten. Sie alle aber sahen, wie sie pathologisch die P. n. als Entzündungsprozeß betrachteten. ätiologisch die P.n. als infektiösen Ursprungs an.

Demgegenüber haben andere Autoren den infektiösen Charakter der P. n. überhaupt zu leugnen versucht und in ihr die Folge einer Störung mechanischer Gleichgewichtskorrelationen gesucht; so P. Meyer, der die P. n. auf eine durch ein auschweifendes Leben bedingte, abnorme Blutdrucksteigerung zurückführte, so Eppinger, der in den periarteritischen Anewrysmen Folgen kongenitaler Debilität der Elastika sah, so Ferrari, der die P.n. als Ernährungsstörung, gleichsam als alimentäre Intoxikation auffaßt: so in nenester Zeit Pertik, der ihr eine kongenitale Debilität der Media zugrunde legt, sowie Benda, der eine mechanische Noxe des Blutdrucks bei Schwäche der GefäBwand infolge allgemeiner Störung der Ernährung oder der Innervation oder der Anlage als Ursache der P. n. substituiert.

Derartige Überlegungen, die die Ursache der P. n. in einer Störung des mechanischen Gleichgewichts der Elemente des Blutkreislaufs suchten, lassen sich im einzelnen zumeist schon durch allereinfachste Überlegungen allgemeinster Art widerlegen. So die Ansicht von P. Meyer, nach der Vermehrungen des Blutdrucks infolge ausschweifenden Lebenswandels bei körperlichen Anstrengungen die P. n. bedingen, durch den Hinweis darauf, daß die P. n. bei kleinen Kindern beobachtet worden ist (s. o.). So die Meinung von Eppinger, nach der die P. n. die Folge einer kongenitalen Debilität der Elastika ist, durch die Tatsache, daß P. n. bei alten Franen am Eingang des Senium beobachtet wurde, wobei es schwer fällt, sich vorzustellen. daß eine kongenitale Schwäche bis zum Eintritt des Senium latent bleiben könne usw. (s. o.).

Dazu kommt aber als wichtigstes Gegenargument die Tatsache, daß es heute nicht mehr möglich ist, eine derart ausgeprägte Entzïndung wie die, die wir im Ablauf der P. n. einsetzen sehen, als Resultat einer einfachen 
Regulationsstörung im Sinne des Ausfalls mechanischer Komponenten aufzufassen.

Wenn sich auch heute die Faktoren, die die Kriterien der Entzündung bedingen, weder in ihrer Gesamtheit, noch in ihren Einzelheiten übersehen lassen, so scheint sich doch allmählich die Erkenntnis herauszubilden, daß es nicht schlechthin jeglicher Zerfall organischer Substanz ist, der die für die akute Entzündung charakteristische Reaktion, im besonderen die Reaktion am Gefäßapparat und darunter wieder besonders die Leukozytose hervorzurufen vermag, sondern nur die Nekrose und ganz besonders diejenige Koagulationsnekrose, die mit einem Freiwerden bakterieller Gifte verbunden ist.

Unter den Theorien, die in der P. n. eine Infektion sahen, fand weiteste Verbreitung der Vorschlag, in der P. n. eine Äußerung der Syphilis zu erblicken. KuBmaul und Maier kamen in erster Linie zu dieser Auffassung aus der Erwägung heraus, daß zwischen den Ausdrucksformen der P. n. und "manchen Bildern von Syphilis" „eine gewisse Ännlichkeit" bestehe, aber doch wohl ans der Gewohnheit, allerlei Unerklärliches fante de mieux.der Syphilis in die Schuhe zu schieben, um so mehr, als gerade die Syphilis an Spätstadien reich ist, die klinisch und pathologisch mit den Kriterien akuter Syphilis nur in lockerstem Zusammenhang stehen.

Unter den Autoren, die für die syphilitische Genese der P. n. eintraten, muß Versè besonders genannt werden. Die Argumente Versés verdienen besonders gewürdigt zu werden, da Versé selbst betonte, daß er nicht aus lockeren Vermutungen heraus, sondern auf Grund histologischer Bilder einen Zusammenhang zwischen der Arteriitis syphilitica cerebralis und der P. n. fundieren wolle. Versé ging dabei einerseits von der Darstellung einzelner Bilder, die sich im Verlauf der P. n. in der Gefäbwand ausbilden, aus, andererseits von der Zusammenfassung des gesamten Prozesses der Erkrankung.

Bezüglich des letzteren behauptete Versé, daß es sich bei der Periarteriitis nodosa um einen an Ort und Stelle primär anftretenden Entzündungsvorgang handle, der zuerst in den äußeren Schichten der Gefäßwand entsteht. Jede der beiden Außenhäute reagieren dabei in der ihr eigentümlichen Weise: die Media durch Degeneration und Nekrose, die Adventitia durch Proliferation; jedenfalls glaubt Versé, daß „der Prozeß von außen nach innen vorwärts schreitet".

Dem gegenüber möchte ich betonen, daß ich nach der Auffassung, die ich selbst mir angeeignet, und, wie ich glaube, ausführlichst begründet habe, doch die $P$. n. nicht als einen von außen nach innen vorwärts schreitenden Prozeß ansehen kann, sondern als einen Prozeß, der, in den zentralen Partien der Gefäßwand einsetzend, gleichsam nach außen und nach innen konzentrisch um sich greift. 
Bez. der Darstellung der Korrelationen, die in einzelnen Bilder der Arteriitis syphilitica und Manifestationen der P. n. verbinden, hat sich Versé unter anderem auf die Falle von Abramow, besonders auf den 2. Fall von Abramow gestützt. Im ganzen sucht Versé dabei zu zeigen, daß auch bei der P. n., ganz so, wie bei der Arteriitis syphilitica, Riesenzellen und käsige Nekrosen beobachtet worden seien, Riesenzellen z. B. von Graf und P. Müller, Nekrosen von P. Müller.

Dazn sei folgendes bemerkt, Graf und P. Müller haben allerdings, wie übrigens auch Chrostek and Weichselbaum Riesenzellen in periarteriitischen Herden gesehen, aber einerseits ganz vereinzelt, andererseits stets nur in der Intima der Gefäßwand, wo derartige Riesenzellen sicherlich auch aus anderen Ursachen als auf syphilitischer Basis entstehen können, z. B. als Fremdkörperriesenzellen auf den Reiz thrombischer Massen hin, so daß man sicherlich nicht daran denken darf, die Bildung von Riesenzellen bei P. n. als eharakteristisches Symptom von Lues za werten.

P. Müller beschreibt allerdings Nekrosen, aber diese Nekrosen lassen sich, wie all die Nekrosen, die bei P. n. beobachtet worden sind, sehr wohl als primäre Gewebsalteration der Muskelzellen der Media, als Endstadium von Nekrobiosen auffassen, sind ohne weiteres durchaus nicht den Nekrosen echter Gummibildungen gleichzusetzen.

Schließlich bringt Versé noch ein drittes Moment bei, das er als „einen ex juvantibus direkt positiven Beweis" der Syphilisätiologie der P. n. erklärt, nämlich, daß in einem Fall (Schmorl), wo die Diagnose aus der Untersuchung exzidierter Knötchen in vivo gestellt werden konnte, die Affektion nach Einleitung einer antisyphilitischen Kur zum Schwinden gebracht wurde. Dies legt, wie ich ohne weiteres zugeben möchte, den Gedanken nahe, die P. n. auf Syphilis zurückzuführen, kann aber durchaus nicht als Beweis aufgefaßt werden, da es doch immerhin möglich wäre, daß die P. n. auch ohne Darreichung von Antilueticis abgeklungen wäre, wie dies wohl in dem vorliegenden Fall eingetreten ist, und wie es sich nach den Angaben der Autoren auch in dem Fall Pertik-Benedikt vollzogen hat, wo die periarteriitische Affektion zurückging, bevor, wie die Autoren ausdrücklichst bemerken, eine antiluetische Kur mit Jodkali-Darreichung angewandt wurde.

Wenn nun einerseits die Momente, die im Sinne einer Ähnlichkeit der P. n. und Arteriitis syphilitica angeführt worden sind, einer Kritik nicht standhalten, so können auf der anderen Seite zahlreiche Punkte angeführt werden, die gegen einen Zusammenhang von Arteritis syphilitica und P. n. sprechen. Und wenn auf Grund der Tatsache, daß dem Organismus doch gleichsam stets nur eine beschränkte Auswahl von Reaktionsmöglichkeiten zur Verfügung steht, eine Übereinstimmung der histologischen Bilder auch bei völliger Verschiedenheit der ätiologischen Momente - man vergleiche z. B. Lepra und 
Tuberknlose, selbst Syphilis und Tuberkulose - zustandekommen kann, dann muß andererseits, gerade angesichts der geringen Anzahl von Reaktionsmöglichkeiten des Organismus, eine Differenz der histologischen Bilder mit eindrucksvollster Deutlichkeit gegen die Annahme einer gemeinsamen Ätiologie von Arteriitis syphilitica und P. n. sprechen.

In der Tat lassen sich nun zwischen den Befunden der P. n. und den pathologisehen Veränderungen, die ron der Arteriitis syphilitica gezeitigt werden, weitgehende Differenzen nachweisen. Derartige Differenzen sind zum ersten Male von Guldner, dem wir die letzte Veröffentlichung über P. n. verdanken, zusammengestellt worden. Guldner führt an:

1. „Bei der syphilitischen Arteriitis sind die Gehirnarterien wohl selten völlig intakt, im Gegensatz zur P. n., die fast nie die Gehirnarterien befällt."

Dazu sei bemerkt, daß de facto nur Chvostek und Weichselbanm und P. Müller eine P.n. an den Gehirnarterien beobachten konnten.

2. "Der Spirochäten-Befund war bei der P. n. stets negativ."

Dies möchte ich dahin ergänzen, daß in nenester Zeit, was Guldner anseheinend noch nicht bekannt war, bei der Arteriitis syphilitica Spirochäten in den Erkrankungsherden nachgewiesen wurden (Benda, Straßmann), hingegen bei der P. $n$. auch in den neuesten Untersuchungen niemals Spirochäten gefunden worden sind.

3. "Gummata und Riesenzellen hat man bei der P. n. nie gesehen."

Dieser letzte Einwand ist, wie ich oben bereits betont habe, nicht mehr völlig åufrechtzuerhalten, insofern Chvostek a. Weichselbaum, Graf P. Mäller tatsächlich Riesenzellen bei P. n. gesehen haben, allerdings stets, nur ganz vereinzelt und stets nur in unmittelbarer Nachbarschaft des Gefäßlumens, sodaß man an eine isolierte und selbständige Bildung der Riesenzellen, vielleicht an eine Bildung von Fremdkörperriesenzellen auf den Reiz thrombischer Massen hin, denken kann.

4. Orth und $\nabla$. Baumgarten gaben an, daB bei der luetisehen Arteriitis die Elastika in ihrer Kontinuität gewöhnlich nieht gesehädigt ist, daß dagegen eine lamellöse Aufsplitterung der elastisehen Elemente zur Exscheinung. kommt, die. im Sinne einer Vermehrung dieser Elemente zu denten sei. Diese Erscheinung stimmt ebenfalls nicht mit dem histologischen Bilde der P. n. überein."

Dieser Einwand von Guldner scheint mir sehr bemerkenswert. Auch ich selbst konnte gerade die Endstadien von P. n. frei von Neubildungen elastischer Elemente finden, während bei der Arteriitis syphilitica, wie alle Autoren betonen, gerade die Endstadien durch eine lamellöse Aufsplitterung der elastischen Elemente charakterisiert sind.

5. "Die Intima verhält sich bei der Lues ebenfalls anders als bei der P. n. Bei dieser erkrankt sie an letzter Stelle, bei der Syphilis hingegen 
wird sie, nach Heubner, schon sehr frühzeitig affiziirt, jedenfalls früher als die Media".

Auch ich möchte betonen, daß die Gefäßwandintima bei der Arteriitis syphilitica schon in einem frühen Stadium Veränderungen zeigt, hingegen bei der P. n. erst erkrankt, wenn die übrigen Gefäßwandschichten schon rom ProzeB ergriffen sind. Tch möchte noch hinzufügen, daß die Media der GeläBwand bei der Arteriitis syphilitica sehr lange resistent bleibt, hingegen bei der P. n. zuerst, jedenfalls schon im Beginn des Prozesses verändert wird.

6. „Die Wassermann-Reaktion, die bisher nur in einzelnen Fällen ansgeführt werden konnte (Löhlein,. Veszprémi, Bloch), ist bei der P.n. noch nicht als positiv befunden worden."

Auch dieser Einwand läßt sich heute nicht mehr schlechthin ausprechen. da in dem rorliegenden Fall von P. n. die Wassermann-Reaktion ein positives Resultat ergab. Doch muß gerade im vorliegenden Fall das Ergebnis der Wassermann-Reaktion als irrelevant angesehen werden, da Patient nicht nur an einer P. n. gelitten hatte, sondern anch an einer von der P. n. völlig unabhängigen und die P. n, völlig unberührt lassenden Syphilis litt, die für den positiven Ausfall der Wassermann-Reaktion verantwortlich gemacht werden muB.

Dafür glaube ich drei weitere und, wie mir scheint, recht wesentliche Differenzen, durch die sich die Arteriitis syphilitica und die P. n. unterscheiden, anführen zu können:

1. Die bei der P. n. auftretenden entzündlichen Infiltrationen sind fast stets ron Leakozyten gebildet, entspreehend dem Umstand, daß auch das Blutbild zumeist von einer ausgesprochenen Leukozytose beherrscht wird. die Infiltrationen, die im Ablauf der Arteriitis syphilitica entstehen, werden vorwiegend von Lymphozyten und Plasmazellen formiert, wie sich auch im Blutbilde der Arteriitis syphilitica niemals eine Leukozytose bemerkbar macht.

2. Aneurysma-Bildungen bilden, sozusagen, eine regelmäBige Komplikation der P. n. und stellen sich in ihren Spätstadien mit einer geradezu charakteristischen Regelmäßigkeit ein, bei der Arteriitis syphilitica werden Aneurysmen nur äuBerst selten (Benda) beobachtet.

3. Die Arteriitis syphilitica spielt sich meist auf der ganzen Zirkumferenz des GefäBquerschnitts $a b$, indem sich die adventitielle Infiltration mantelförmig über die Media ausbreitet (B enda), bei der P. n. ist aber nur ein Teil, meist nur ein sehr geringer Teil der Getäßzirkumferenz ergriffen.

SchlieBlich läßt sich noch ein Punkt anführen, der geeignet ist, die Annahme der Syphilis-Genese der P. n. zum mindestens in Frage zu stellen: nämlich die Tatsache, daß bei Tieren, in größter Ausdehnung bei Axishirschen, Erkrankangen beobachtet worden sind, deren pathologische Bilder dem Bilde der P. n. sicherlich ähnlich, wahrscheinlich wesensgleich sind. 
Lüpcke sah (1906) bei Axishirschen eine Epidemie auftreten, in der die Tiere teils in Kürze unter den Erscheinungen einer schweren Entzündung des Magendarmtraktus, teils in protrahiertem Verlauf unter den Symptomen allgemeiner Macies und allgemeiner Asthenie zugrunde gingen. Autoptiseh zeigten die Tiere neben Veränderungen eines Marasmus und einer universellen Anämie vor allem Veränderungen der Gefäße; speziell der kleinen Arterien, und zwar knotige Verdickung der Wandung mit Verengerung oder VerschluB des Lumens, teilweise mit Aneurysmen und mit Thrombosen.

Histologische Untersuchungen wurden von Jäger vorgenommen. Sie zeigten eine gewaltige Infiltration resp. Granulation innerhalb der Adventitia und Zerstörung der Media durch Eindringen derartiger Granulationen. Jäger selbst schlob aus seinen Untersuchungen, daß es sich um P. n. bei Tieren handle, und daß wohl für die P. n. der Tiere und der Mensehen dieselbe Noxe, nämlich ein Infektionserreger, in Betracht komme.

Diese Anschaungen $J$ ägers wurden versehiedentlich in Frage gestellt, vor allem von Mönckeberg, der betonte, daß eine Erkrankung, die mit einer Infiltration der Adventitia beginne, nicht mit der menschlichen P. n. identifiziert werden dürfe, da für diese eine primäre Nekrose der Media vor allem charakteristisch sei. Ich selbst möchte mir aus der Beschreibung des Bildes keinen definitiven Schluß gestatten. Immerhin kann ich mir wohl vorstellen, daß die Axishirsche de facto von P. n. ergriffen werden, daß aber bei ihnen, vielleicht infolge der starken Reaktionsfähigkeit tieriseher Organismen, die Reaktionserscheinungen seitens der Adventitia gegenüber den Alterationserscheinungen der Media derart überwiegen, daß die letzteren gleichsam darunter verschwinden, anders ausgedrückt, daß die sekundären Proliferationserscheinungen der Adventitia die Media schon überwnchert haben, bevor die primären Alterationen der Media überhaupt sichtbar gemacht werden können.

Dieser Gedanke scheint mir insofern einer Begründung nicht zu entbehren, als auch am Schwein (Joest) und am Hausrind (Guldner) Beobachtnngen einer Erkrankung erhoben wurden, die einerseits der menschlichen P. n. überraschend ähnlich sah, andererseits sich ebenfalls dadureh ron der menschlichen P. n. unterschied, dab die Reaktionserscheinungen der Adventitia gegenüber den Alterationserscheinungen der Media zeitlich und räumlich im Vordergrund standen.

Wenn aber die "Axisseuche" wirklich als P. n. aufzufassen ist, dann kann die Syphilis fernerhin nieht mehr für die Genese der P. n. in Anspruch genommen werden.

Wie dem aber auch sei, es seheint mir nicht angängig zu sein, die Syphilis als Ursache der P. n. anzusehen. Denn im ganzen können wir über die Prozesse, die sich einerseits bei der P. n., andererseits bei der Arteriitis syphilitica abspielen, sagen: bei der P. $n$. handelt es sich um eine 
banale Entzündung in der Media der Gefäßwand, bei der Arteriitis syphilitica um eine Ausbildung spezifischer Entzündungsprodukte in den äußeren Gefäßwandschichten. -

Auch weiterhin gibt es nichts, was darauf hinwiese, irgendeinen Erreger, der zurzeit bekannt wäre, zur Erklärung der Genese der P. n. anzuführen. Zwar haben einzelne Autoren bakterielle Befunde notieren können: so beschrieb Beitzke einen Fall, an dem er in den periarteriitischen Herden Streptokokken fand, faßte aber selbst die Streptokokkenbefunde als Ausdruck einer Mischinfektion auf. So haben auch von Bomhard-Oberndorffer in einem Fall Staphylokokken sichtbar machen können und haben, da der Fall auch die klinischen Symptome einer Staphylokokkensepsis aufwies, darin einen symptomatischen Befund sehen wollen; sie glaubten als Erreger der P. n. den Staphylokokkus bezichtigen zu dürfen, eine Folgerung, die aber wegen der völligen Isoliertheit, in der sie steht, später nicht mehr aufgegriffen wurde, und die wohl nicht als eine Grundlage der Atiologie der P. n. gelten kann.

Es bliebe noch die Möglichkeit, die P. n. auf ein "unbekanntes Virus" zurückzuführen.

$\mathrm{Zu}$ der Anschauung, in der P. n. eine Infektionskrankheit unbekannten Ursprungs, aber doch eine Infektionskrankheit sui generis zo sehen, wäre man aber angesichts des völligen Fehlens von bakteriologischen Befunden und von Ergebnissen des Tierexperiments nur dam berechtigt, wenn es gelänge, innerhalb der klinischen Symptome oder innerhalb der pathologischen Befunde eine derartig eindentige, regelmäßig zu konstatierende Eigenart nachzuweisen, daß es gleichsam der Logik primitiver Uberlegungen widerspräche. einer solchen Einheitlichkeit des Ablaufs Differenzen des Ursprungs zu untersehieben.

Es bleibt also die Aufgabe, sowohl die klinischen Angaben als auch die pathologischen Notizen, die bez. der P. n. vorliegen, einerseits anf die Eigenart der Symptome und Befunde zu prüfen.

Bez. der Klinik der P. n. gelangt man dabei, wie schon früher angeführt wurde, wohl zu folgendem Resultat: es finden sich bei P. n. allerdings einige Symptome, die innerhalb der Mehrzahl der Fälle mit größter Regelmäßigkeit wiederkehren, z. B. die Leukozytose, das Fieber, die Milzschwellung, in gewissem Maße auch die Albuminurie und die Symptome der Herzschwäche. Derartige Symptome entbehren aber aller Eigenart; denn Fieber, Milzschwellong, ferner Nierenschädigung und Herzschwäche können jegliche Infektionskrankheit begleiten.

Im übrigen sind die Symptome der P. n. zumeist abhängig von den Folgen der periarteriitischen Veränderungen.

Schließlich kommen Fälle von P. n. vor, in denen die Krankheit gar nicht in Erscheinung tritt, sondern sich gewissermaßen gänzlich unter den 
Bildern einer andern Erkrankung verbirgt, wie z. B. unter dem Bilde einer Streptokokken-Sepsis (Beitzke), einer Skarlatina (Hart-Künne), eines Morbus maculosus Werlhoffii (Zimmermann), einer Polyneuritis (Lorenz), einer Angina mit Staphylokokken-Infektion (v. Bomhard-Obernd orffer) usw.

Und im allgemeinen zeigte jeder Fall andersartige Symptome und baute sich auf einer eigenartigen Anamnese auf.

Ähnlich sind die Ergebnisse, die sich bei der Durchsicht der Pathologie der P. n. für das Problem der Eigenart der Erkrankung gewinnen lassen.

Der Prozeß läBt sich letzten Endes wohl so definieren: Eine Entzün. dung der Mittelschicht kleiner Arterien, aus der heraus es durch reaktive Beteiligung der Innenschicht und der Außenschicht zur Entwicklung zirkumskripter Gefäßwandnarben kommt, alias eine banale, in ihrem Ablauf und in ihrem Ergebnis indifferente "Mesarteriitis".

Wenn man aber die Literatur, die über die Erkrankung der kleinen Arterien, speziell bei Infektionskrankheiten vorliegt, durchsieht, so stellt man fest, daß sich bei zahlreichen Infektionskrankheiten in den kleinen Arterien, in größter Regelmäßigkeit und in ausgeprägtestem Maße in den Koronararterien, Prozesse abspielen, die dem Prozeß der P. n. zum mindesten vergleichbar sind.

Solche Veränderungen sind wohl zuerst von Eichhorst beschrieben worden: Eichhorst fand, daßis sich im Ablauf von Infektionskrankheiten oftmals Thrombosen peripherer Arterien entwickeln, und daß an den Stellen der Thrombosen in der Wandung der Arterien pathologische Veränderungen nachzuweisen sind, die er zuerst als endarteriitische Prozesse beschrieb, später als Entzündungen der Adventitia und der Media, letztere bestehend aus einer von der Adventitia gegen die Media vordringenden Infiltration.

Diese "Eichhorstschen Affektionen" sind von Hart und Künne als der P. n. wesensgleich angesehen worden. Meines Erachtens bleibt es unbenommen, derartige Veränderungen der P. n. als vergleichbar zu bezeichnen. Immerhin ist es für mein Empfinden doch nicht angängig, die "Eichhorstschen Affektionen" kleiner Arterien und die Veränderungen der P. n. einander derart zur Seite zn stellen, daß man ans der Genese der ersteren einen Rückschluß auf die Entstehung der letzteren ziehen dürfte. Hingegen sind von Wiesel und von v. Wiesner Affektionen kleiner Arterien, speziell der Koronararterien, mit größter Exalktheit und größter Regelmäßigkeit beschrieben worden, die, nach meinem Dafürhalten, der P. n. geradezu wesensgleich sind.

Wiesel beschrieb diese Veränderungen folgendermaßen: „sie bestehen zunächst in einer herdförmigen Auflockerung des Gewebes der tiefen Mediaschichten, wobei zugleich eine seröse Durchtränkung dieses Gefäßabschnittes stattzufinden scheint. Solche Stellen repräsentieren sich dann als hellere 
Flecken in der Gefäßwand; die Muskelfasern und das spärliche elastische Gewebe solcher Stellen erscheint auseinander gerückt, und zwischen den Fasern liegt die erwähnte, homogen erscheinende Substanz. "In späteren Stadien kommt es nun zunächst zur schlechteren Färbbarkeit der Muskelfasern, sowie zur Quellung der Fasern selbt, die dann noch später zu vollständigem Schwund der Muskulatur and des elastischen Gewebes in solchen Herden führt. Jetzt liegt die interzelluläre Grundsubstanz wie auspräpariert bloß, während Muskulatur und elastisches Gewebe vollständig fehlt. Gewöhnlich aber hat der ProzeB nicht wie bei anderen Arterien an der Elastica interna Halt gemacht, sondern mit der fortschreitenden Auflösung greift die Erkrankung nach Zerstörung der entsprechenden Abschnitte der Elastica interna auch auf die Intima über und führt auch hier zu schwerer Gewebsdegeneration, bis zur Nekrose. An solchen Stellen erscheint dann die Gefäßwand eingesunken; die nekrotischen Partien schimmern grauweiß durch die noch intakten, dem Gefäßlumen zugekehrten Intimaschichten; welcher Vorgang, wie schon erwähnt, makroskopisch häufig zu erkennen ist. Die Nekrose bedeutet den Höhepunkt der Erkrankung, jetzt beginnen sekundäre Prozesse.

Jene Stelle, die nekrotisch wurde, setzt sich scharf von ihrer Umgebung ab, da anscheinend normales Gewebe bis dicht an den Herd reicht. In noch späteren Stadien, mehrere Wochen oder Monate nach abgelaufener Erkrankung, zeigt sich, daß die nekrotischen Stellen mit Bindegewebe durchwachsen sind. Es ist zur Bildung einer mesarteriitischen resp. endarteriitischen Narbe gekommen."

Im übrigen beschreibt Wiesel noch Veränderungen, die als Übergänge solcher mesarteriitischen Narben zu atherosklerotischen Schwielen aufgefaßt werden können, und von denen aus er zn dem Ergebnis kommt; „die akuten Infektionskrankheiten für eine wichtige Ursache späterer Koronar-Sklerosen" halten zu müssen.

Solche Veränderungen wurden von Wiesel bei Scharlach, Masern, Diphtherie und akuten eitrigen Prozessen beobachtet, später aber von v. Wiesner auch bei Endokarditis, Pyämie, andern Infektionsprozessen, vor allem „bei Personen mit erworbener Syphilis" in den Kranzgefäßen gefunden.

Sie sind allerdings, sowohl von Wiesel als auch $v$. Wiesner, nur in größter Kürze beschrieben worden und haben auch sonst, soweit ich übersehen kann, in der Literatur keine Darstellung mehr gefunden.

Im übrigen sind sie von Wiesel und v. Wiesner. selbst weder einer Nomenklatur unterworfen noch in ein System eingereiht worden. Wo sie späterhin erwähnt worden sind, sind sie als "Nekrosen", d. h. als rein regressive Metamorphosen betrachtet worden (Benda, Hart.)

Demgegenüber möchte ich betonen: wenn die Wiesel-Wiesnerschen Affektionen, wie erwähnt, schließlich in Narbenbildungen übergehen, so muß man annehmen, daB auf die primäre Nekrose entsprechend dem Organisations- 
prozeß bei anderen derartigen Veränderungen (Infarkten usw.) ein Stadium der Exsudation. und der Proliferation folgt, daß also eine, wenn auch nur schleichend verlaufende Entzündung; die Wiesel-Wiesnerschen Affektionen bedingt.

Darnach ist es klar, daß die Wiesel-Wiesnerschen Affektionen und die P. n. vergleichbare Prozesse darstellen: hier wie dort primäre Nekrosen der Muskelschicht kleiner Arterien mit Exsudation und Proliferation und mit Ausgang in Narbenbildung. Es ist darnach auch klar, daß die P. $n$. weder bezüglich der Klinik noch bezüglich der Pathologie eine derartige Eigenart besitzt, daß man berechtigt wäre, ihr bloß in Anbetracht der nur graduellen Unterschiede gegenüber den Wiesel-Wiesnerschen Veränderungen eine Sonderstellung der Ätiologie zuzuweisen: d. h. man hat kein Recht, einen „unbekannten Erreger" zu substituieren, um die Entstehung der P. n. zu erklären.

Damit bleibt nur noch die Möglichkeit übrig, anzunehmen, daß verschiedene Erreger an der Auslösung der P. n. beteiligt sein können (HartKünne, G. B. Gruber).

Màn gelangt also bezüglich der Ätiologie der P. n. zu dem Schluß, daß es sich um eine pathologisch einheitlich charakterisierte, aber ätiologisch versehiedentlich bedingte Erkrankung handelt.

$\mathrm{V}$

Schon ältere Autoren haben die P. n. in Parallele gesetzt zu den WieselWiesnerschen Affektionen, vor allem bezüglich der Atiologie, indem sie glaubten, beide Veränderungen als Folgeerscheinungen verschiedener Infektionen ansehen zu dürfen. Ich möchte einen Schritt weiter gehen und sagen, daß die Wiesel-Wiesnerschen Veränderungen der P. n. nicht nur vergleichbar sind, sondern mit ihr völlig übereinstimmen, daß beide Affektionen nur graduelle, nicht aber qualitative Differenzen aufweisen.

Ich habe darauf hingewiesen, daß das Endstadium der Wiesel-Wiesnerschen Affektionen, die "Medianarbe", die Annahme einer Entzündung im Verlaufe des Prozesses postuliert, ich habe aber auch gerade aus dem vorliegenden Falle im Vergleich mit den früher publizierten Fällen von P. $\mathbf{n}$. den Schluß gezogen, daß die P. n. ebenfalls auf dem Wege einer banalen Entzündung in "Medianarben" ausgeht. Um die Identität der beiden in ihrem Beginn, der Medianekrose und in ihrem Ansgang, der Medianarbe, identischen Prozesse festzustellen, bleibt also nur noch übrig, nachznweisen, daß tatsächlich Übergangsformen zwischen den beiden Erkrankungen vorkommen, bei denen man zweifelhaft sein kann, welcher von beiden man sie zurechnen soll.

Als solche Übergangsformen kann man aber meines Erachtens einerseits 
den Fall von $H$ art und $K \ddot{u} n n e$, andererseits den vorliegenden Fall ansehen:

Den Fall von Hart-Künne insofern, als bei ihm im grobanatomischen Bilde die Erscheinungen der P. n. völlig fehlten und nur mikroskopische Veränderungen nachzuweisen waren, - ganz wie bei den Affektionen von Wiesel und v. Wiesner; den vorliegenden Fall aber insofern, als bei ihm keinerlei klinische Symptome anfgetreten waren und sich nur anatomische Prozesse ausgebildet hatten -, also auch gewissermaßen im gleichen Sinne, wie bei den Affektionen von Wiesel und v. Wiesner.

Darnach ließe sich denken, daß es auch Fälle von P. n. gibt, die sowohl der klinischen als auch der autoptischen Betrachtung verborgen bleiben, sowohl den Blicken der Ärzte, als anch der Pathologen entgehen können, also ganz im Sinne der Wiesel-Wiesnersehen Affektionen auftreten.

Und daß es auch derartige Fälle, derartige typische Utbergänge zwischen Wiesel-Wiesnerschen Affektionen und P. n. gibt, dafür glaube ich den Beweis nun tatsächlich erbringen zu können:

Am 25. Mai 1917 wurde im Pathologischen Institut zu Straßburg eine 63 jährige Frau obduziert, die unter der Diagnose „Inanition-BronchitisGastritis (Scirrhus ventriculi?)" eingeliefert worden war.

Die Autopsie (Prof. Dr. Mönckeberg) ergab: Große weiße Nieren mit Hypertrophie des linken Ventrikels. Emphysem mit chronischer eitriger Bronchitis und exzentrischer Hypertrophie des rechten Ventrikels. Chronischer Magenkatarrh. Allgemeine Atrophie. Pleuraadhäsionen beiderseits, Ödem der weichen Hirnhäute. Massige Atherosklerose.

Bei der mikroskopischen Untersuchung der Nieren zeigte sich, das neben einer interstitiellen Nephritis in Form einer Periglomerulitis eigentümliche Veränderungen der kleinen Arterien der Nieren vorlagen: teils Nekrosen der Media in Verbindung mit Infiltrationen der Adventitia und Proliferationen der Intima; teils Umwandlungen der Gefäßwand und des Gefäßlumens in ein derbes, gleichmäBiges Narbengewebe, in dem noch Bruchstücke elastischer Lamellen enthalten sind, teils auch Aneurysmabildungen und in Organisation begriffene extramurale Hämatome. D. h. es fanden sich hier Bilder, die nach jeder Richtung hin als Bilder einer P. n., sei es akuter, sei es abgelaufener Stadien gedeutet werden mußten, ohne daß in der Klinik oder bei derAutopsie ein Moment beobachtet worden wäre, daß für das Vorhandensein dieser Erkrankung gesprochen hätte.

Damit wäre wohl bewiesen, daß zwischen beiden Formen der "Mesarteriitis", den Wiesel-Wiesnerschen Affektionen und der KuBmaulMaierschen P. n. Ubergänge vorkommen.

Wenn aber zwischen den mesarteriitischen Affektionen, die von Wiesel und v. Wiesner beschrieben wurden, and den mesarteritischen Affektionen, die unter der Bezeichnung Periarteriitis nodosa zuerst von KuBmaul und 
Maier veröffentlicht worden sind, nur ein gradueller Unterschied besteht, so kommt man schließlich $\mathrm{zu}$ folgendem Schluß:

Es gibt keine Periarteriitis nodosa, wenigstens nicht in dem Sinne, in dem die Bezeichnung bisher gebraucht wurde.

Denn die Periarteriitis nodosa ist überhaupt keine Erkrankung sui generis. Die Erkrankungen, die bisher als P.n. bezeichnet wurden, gehören in die Reihe der Prozesse von "Mesarteriitis", die sich nach jeglicher Infektion einstellen können.

Sie ist vielmehr als solehe Mesarteritis nur eine Begleiterscheinung von Iufektionskrankheiten etwa im Sinne der Myokarditis oder der Endokarditis oder der Nephritis. . . . .

\section{VI.}

Es bleibt schließlich noch die Frage $\mathrm{zu}$ beantworten, weshalb sich innerhalb der zahlreichen Fälle von Mesarteriitis hie und da einzelne Fälle von besonders schwerem, sozusagen "foudroyantem" Verlaufe entwickeln.

Wie mir scheint, kommt hierbei wahrscheinlich eine abnorme Disposition in Betracht, etwa im Sinne einer kongenitalen Schwäche des Gefäßsystems (Eppinger, Hart, Benda), speziell der Muskelschicht des Gefäßsystems (Benda) oder im Sinne einer Steigerung des Blutdrucks vielleicht infolge von Anstrengungen und Ausschweifungen (P. Meyer), vielleicht auch im Sinne neuropathischer Störungen (Ferrari).

Es ist vielleicht auch angesichts der Versehiedenheit der Erreger, nach deren Invasion „mesarteriitische" Prozesse auftreten, anzunehmen, daß die Noxen, die die "Mesarteriitis" erzeugen, nicht exogener Natur, sondern endogenen Ursprungs sind, gleichsam Autointoxikationen darstellen. Und es bleibt die Möglichkeit, die quantitativen Prominenzen einzelner Fälle von Mesarteriitis durch eine quantitative Prominenz solcher AutointoxikationsProdukte, infolge quantitativ abnormer Reaktionsfähigkeit des Organismus zu erklären. . . . . .

Immerhin gehört dies ins Reich der Hypothese.

\section{Literatur.}

Abramow: Uber Veränderungen der Blutgefäße bei der Syphilis. Zieglers Beitr. für path. Anat, u. allg. Path. Bd. 26. - Aschoff: Diskussion zu Lüpkes Vortrag über Periarteritis nodosa. Verh. d. D. Path. Ges., X. Tagung, 1906. - Beitzke: Präparate eines Falles von Periarteritits nodosa. Berl. klin. Wschr. 1381., 1908. - Derselbe: Diskussion zu Jägers Vortrag über Periarteriitis nodosa. Verh. d. D. Path. Ges., XUI. Tagung, 1909 - Derselbe: Uber einen Fall von Periarteriitis nodosa. Virch. Arch. 199. - Benda: Ũber die sogenannte Periarteritis nodosa. Berl. klin. Wschr., 353, 1908. - Derselbe: Aneurysma mach Syphilis. 'Verh. d. D. Path. Ges., VI. Tagung, 1904. - Ders elbe: Diskussion zu Jägers Vortrag über Periarteritis nodosa. Verh. d. D. Path. Ges., XIII. Tagung, 1909. - 
Derselbe: Die GefäBe. Aschoffs Lehrbuch der path. Anat. - Benedict: Uber Periarteritis nodosa. Ztschr. 1 . klin. Med, 64, 1907. - Bloch, Vera: Uber Periarteritis nodosa. L.-Diss. Zürich 1913. - - Bomhard: Periarteriitis nodosa, als Folge einer Staphylokokkensepsis nach Angina. Virch. Arch. 192. - Datnowski: Uber Periarteriitis nodosa. 1.-Diss., Berlin 1909. - Dickson: W. E. Carnegie: Unique case of polyarteritis acuta nodosa. The Lancet S. 226, 27. I. 1907. Zitiert nach G. B. Gruber. - Eichhorst: D. Arch. f. kJin. Med. Bd. 70 u. Bd. 80. - Eppinger: Pathogenese, Histogenese und Ätiologie der Aneurysmen. Arch. f. kI. Ohir. 35. Suppl. 1887. - Ferrari: Über Polyarteriitis acuta nodosa und ihre Beziehungen zur Polymyosytis und Polyneuritis acuta. Zieglers Beitr. $z$. path. Anat. u. allg. Path. 34. - Fletcher; Morley: Uber die sogenannte Periarteriitis nodosa. Zieglers Beitr. z. path. Anat. a. allo. Path 11. - Freand: Zur Kenntnis der Periarteritis nodosa. D. Arch. f. klin. Med. 62. - v. Gierke: Diskussion zu Lüpkes Vortrag über Periarteriitis nodosa. Verh. d. D. Path. Ges., X., 1906. - Graf: Uber einen Fall von Periarteritis nodosa mit multipler Aneurysmenbildung, Zieglers Beitr. $z$. path. Anat. u. allg. Path. 19. - Gruber, G. B.: Uber die Pathologie der Periarteritis nodosa (KuBmaul-Maier). Ztlbl. f. Herza. GefäBkrankheiten, Bd. IX, 1917. - Guldner: Zwei neue Beobachtungen von Periarteritis nodosa beim Nenschen und beim Hausrind. Virch. Archiv, 219 u. L.-Diss., Straßburg 1915. - Hart: Die Mesoperiarteriitis (Peharteriitis nodosa). Berl. klin. Wschr. S. 1305, 1908. - Jäger: Vergleichende pathologische Untersuchungen über die Periarteriitis nodosa. Verh. d. D. Path. Ges., XIII. Tagung, 1909. - v. Kahlden: Ober Periarteriitis nodosa. Zieglers Beitr. z. path. Anat. u. allg. Path. 15. - Derselbe: Referat der Arbeit von Abramow. Ztlbl. f. allg. Path. u. path. Anat, Bd. 12, S. 511. - Krzyszkowski: Przeglad Lekarski 1899 (zit. bei Ferrari, Schreiber, Versé, Gruber). - KuBmaul u. K. Maier: Utber eine bisher nicht beschriebene eigentümliche Arterienerkrankung, dje mit Morbus Brighti und rapid fortschreitender allgemeiner Muskellähmung einhergeht (Periarteriitis nodosa). D. Arch. f. klin. Med. Bd. 1. - Künne: Ober Mesoperiarteritis, - Löhlein: Uber die Bedeutung dex Wassermann sehen Reaktion an der Leiche. Verh.d.D. Path. Ges., XIII. Tagung, 1909. - L arenz: Beitrag zur Kenntnis der multiplen degen. Neuritis. Ztschr. f. klin. Med., 18. - Lüpke: Uber Periarteritis nodosa bei Axishirschen. Verh. d. D. Path. Ges., X. Tagung, 1906. - Marchand: Diskussion zum Vortag Lüplkes über Periarterititis nodosa. Verh. d. D. Path. Ges.. X. Tagung, 1906. - Meyer, P.; Über Periarterititis nodosa usw., Virch. Arch., 74. - Mönckeberg: Uber Periarteritis nodosa. Zieglers Beitr. z. path. Anat. u. allg. Path., 38. - Derselbe: Diskussion zu Jägers Vortrag über Periarteriitis nodosa. Verh. d. D. Path. Ges., XIII. Tagung, 1908. - P. Mïller: Uber Periarteritis nodosa. Festschr. z. Feier d. 50 jahrigen Bestehens des Stadtkrankenhauses zu Dresden-Friedrichstadt. Dresden 1899. - Oberndorffer: Fin Fall von Periarteriitis nodosa. Münch. med. Wschr., S. 2618, 1907. - Derselbe: Diskussion zu Jägers Tortrag über Periarteritis nodosa. Verh. d. D. Path. Ges., XIII. Tagang, 1909. - Pertik: Uber Periarteriitis nodosa. Ref. im Ztbl. f. allg. Path. u. path. Anat, 1908, S. 90, - Ros enGath: Ein seltener Fall von Erkrankung der kleinen Arterien nsw. Ztschr. 1 . klin. Med. Schmidt, J. E.: Uber Periarterïtis nodosa. Zieglers Beitr. z. path. Anat. n. allg. Path. 43. - Schmorl: Diskussion zn Bendas Vortrag uber Aneurysma und Syphilis. Verh. d. D. Path. Ges., VI. Tagung, 1903. - Schreiber: Uber Polyarteritis nodosa. T.-Diss., Köngsberg 1904. - Versé: Uber Periarteriitis nodosa. Münch. med. Wschr, 1905. - Derselbe: Uber Periarteritis nodosa und Arteriitis syphilitica cerebralis. Zieglers Beitx. z. path. Anat. u. allg. Path., 40. - Derselbe: Diskussion zu Jägers Vortrag über Periarteriitis nodosa. Verh. d. D. P. Ges. XIII. Tagang, 1909. - Wiesner: Veränderungen der Koronararterien bei Infektionskrankheiten. Wien. klin. Wschr., 1906, S. 723. - Wies el: Veränderungen der Koronararterien bei Infektionskrankheiten. Wien. klin. Wschr., S. 726. - Veszprémi n. Jankso: Uber einen Fall von Periarteriitis nodosa. Zieglers Beitr. $z$ path. Anat. $u$. allg. Path. 34. - Vesz prémi: Dber Periarteritis nodosa. Zieglers Beitr. z. pat. Anat. a. allg. Path. Bd. 52. Weichselbanm n. Chvostek: Herdweise syphilitische Endarteritis mit multipler Aneurysmabildung. Wien. med. Ztg. 1877. - Zimmermann: Wagners Arch. f. Heilk. 1874 (zitier bei Versé, Ferrari. G. B. Gruber). 